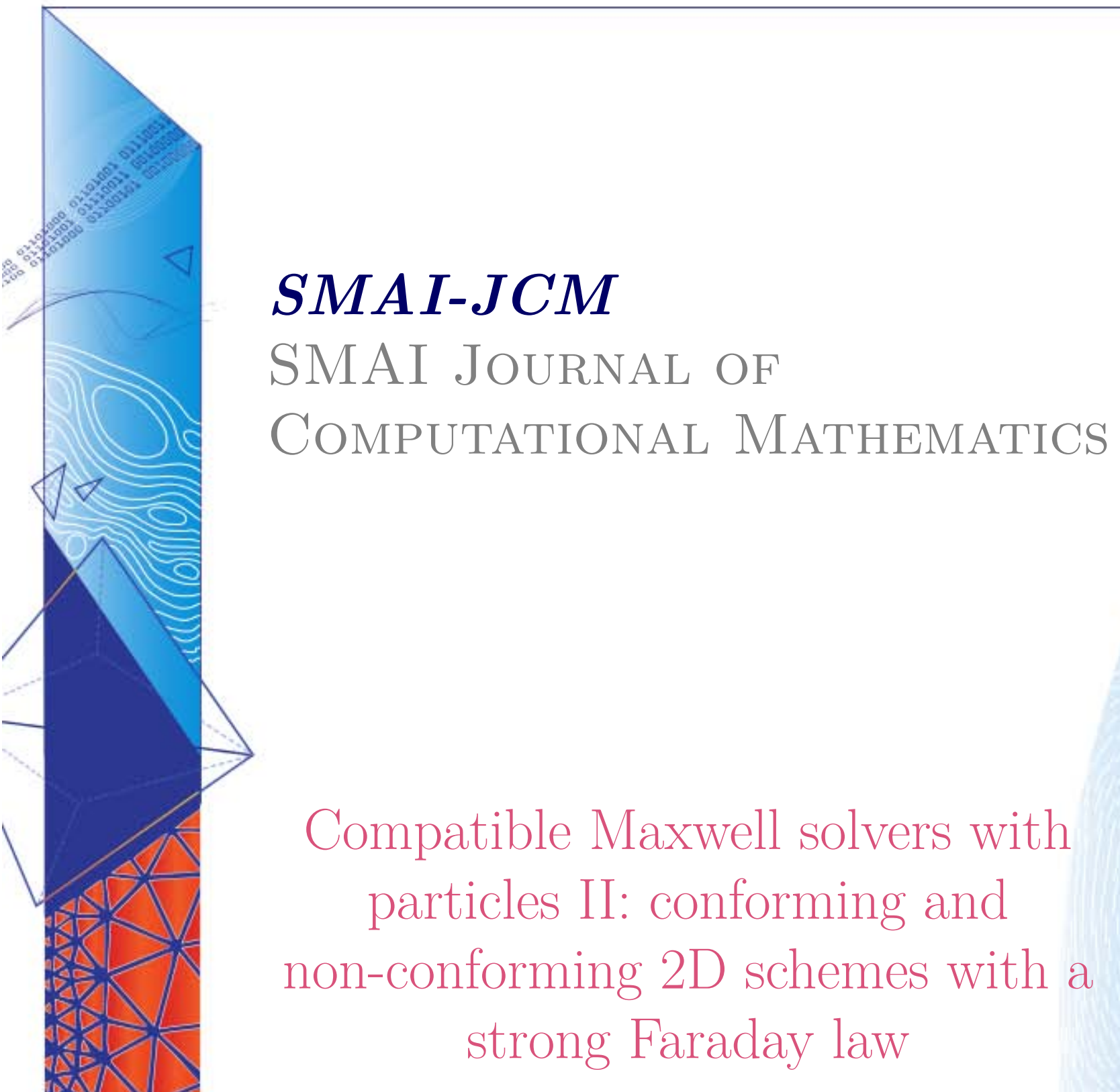

\author{
Martin Campos Pinto \& Eric Sonnendrücker \\ Volume 3 (2017), p. 91-116. \\ $<$ http://smai-jcm.cedram.org/item?id=SMAI-JCM_2017__3__91_0> \\ ๑ Société de Mathématiques Appliquées et Industrielles, 2017 \\ Certains droits réservés.
}

\begin{abstract}
cedram
Article mis en ligne dans le cadre du

Centre de diffusion des revues académiques de mathématiques

http://www.cedram.org/
\end{abstract}
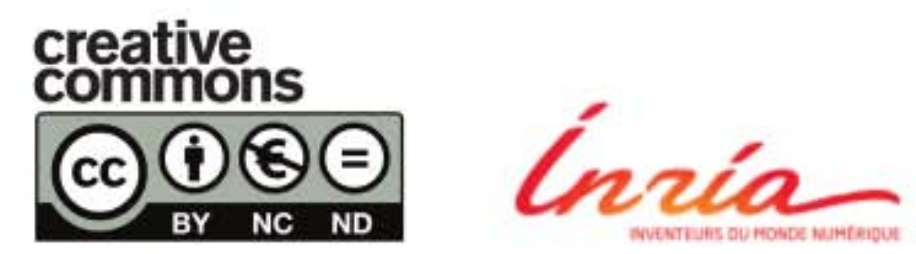


\title{
Compatible Maxwell solvers with particles II: conforming and non-conforming 2D schemes with a strong Faraday law
}

\author{
Martin Campos Pinto ${ }^{1}$ \\ ERIC SONNENDRÜCKER ${ }^{2}$
}

\footnotetext{
${ }^{1}$ CNRS, Sorbonne Universités, UPMC Univ Paris 06, UMR 7598, Laboratoire Jacques-Louis Lions, 4, place Jussieu 75005, Paris, France

E-mail address: campos@ljll.math.upmc.fr

2 Max-Planck Institute for plasma physics, Boltzmannstr. 2, D-85748 Garching, Germany, Mathematics Center, TU Munich, Boltzmannstr. 3, D-85747 Garching, Germany

E-mail address: sonnen@ipp.mpg.de.
}

\begin{abstract}
This article is the second of a series where we develop and analyze structure-preserving finite element discretizations for the time-dependent 2D Maxwell system with long-time stability properties, and propose a charge-conserving deposition scheme to extend the stability properties in the case where the current source is provided by a particle method. The schemes proposed here derive from a previous study where a generalized commuting diagram was identified as an abstract compatibility criterion in the design of stable schemes for the Maxwell system alone, and applied to build a series of conforming and non-conforming schemes in the 3D case. Here the theory is extended to account for approximate sources, and specific chargeconserving schemes are provided for the $2 \mathrm{D}$ case. In this second article we study two schemes which include a strong discretization of the Faraday law. The first one is based on a standard conforming mixed finite element discretization and the long-time stability is ensured by the natural $L^{2}$ projection for the current, also standard. The second one is a new non-conforming variant where the numerical fields are sought in fully discontinuous spaces. In this $2 \mathrm{D}$ setting it is shown that the associated discrete curl operator coincides with that of a classical DG formulation with centered fluxes, and our analysis shows that a non-standard current approximation operator must be used to yield a charge-conserving scheme with long-time stability properties, while retaining the local nature of $L^{2}$ projections in discontinuous spaces. Numerical experiments involving Maxwell and Maxwell-Vlasov problems are then provided to validate the stability of the proposed methods.
\end{abstract}

Math. classification. 35Q61, 65M12, 65M60, 65M75.

Keywords. Maxwell equations, Gauss laws, structure-preserving, PIC, charge-conserving current deposition, conforming finite elements, discontinuous Galerkin, Conga method.

\section{Introduction}

Like its companion article [10], this work addresses the issue of long-time stability in time-dependent Maxwell solvers, either considered alone or coupled with an additional scheme for the current sources. It is known that this issue is strongly related to the good preservation of the divergence constraints at the discrete level. We refer to the introduction of [10] for a review of the literature on which our work is based, and for a presentation of its main guiding lines.

In this article we pursue this study and propose two compatible schemes that include discrete Faraday laws in strong form for the 2D Maxwell system

$$
\left\{\begin{array}{c}
\partial_{t} B+\operatorname{curl} \boldsymbol{E}=0 \\
\partial_{t} \boldsymbol{E}-c^{2} \operatorname{curl} B=-\frac{1}{\varepsilon_{0}} \boldsymbol{J} .
\end{array}\right.
$$

The first scheme is a standard curl-conforming mixed finite element method for which we verify that a standard Galerkin $\left(L^{2}\right)$ projection provides a Gauss-compatible approximation for the current $\boldsymbol{J}$, 


\section{Campos Pinto \& E. Sonnendrücker}

in the sense of [11]. The second scheme extends this construction to spaces of fully discontinuous fields as in standard DG methods, in order to avoid inverting global mass matrices. It belongs to the class of conforming/non-conforming (Conga) methods designed in [11] to preserve the mixed form of conforming Galerkin approximations, and it also comes with a compatible approximation operator for the current. An interesting feature of this particular method is that, in $2 \mathrm{D}$, the associated discrete curl operator coincides with that of a classical DG method with centered fluxes. However, the current approximation operator is non-standard.

We then complete these two schemes by identifying for each of them the discrete divergence operators that form a complete structure-preserving discretizations in the sense of [10]. In the case where the Maxwell system (1.1) is coupled with an additional equation for the source, such as a Vlasov equation

$$
\partial_{t} f+\boldsymbol{v} \cdot \nabla_{x} f+\frac{q}{m}(\boldsymbol{E}+\boldsymbol{v} \times B) \cdot \boldsymbol{\nabla}_{\boldsymbol{v}} f=0
$$

describing the collisionless evolution of one or several particle species with charge $q$ and mass $m$ through their distribution function $f=f(t, \boldsymbol{x}, \boldsymbol{v})$, this framework allows us to show that the approximation operators identified as Gauss-compatible for the Maxwell system alone can also be used to deposit the associated current density

$$
\boldsymbol{J}:=\int \boldsymbol{v} f \mathrm{~d} \boldsymbol{v}
$$

(or better, its approximation by numerical particles) on the finite element spaces in a stable, chargeconserving way, be it for the conforming or the non-conforming Galerkin discretization.

Finally, we provide numerical experiments that validate the approach and the numerical convergence of the proposed schemes (established by theoretical means for the Maxwell system alone), using a pure Maxwell problem and an academic Maxwell-Vlasov test case.

The outline is as follows: In Section 2 we briefly recall the main criterion identified by the stability analysis developped in our previous works $[11,10]$ to the case considered here of a 2D Maxwell equations with a strong Faraday law. Then in Section 3 we introduce the needed discrete function spaces for a conforming Finite Element approximation and verify that they fit into our abstract framework. We next consider in Section 4 the case of discontinuous non conforming Finite Elements where our framework enables in a non trivial way to construct long time stable discretizations. We also show that in 2D, this new scheme can be formulated as a Discontinuous Galerkin (DG) scheme with a non-standard current approximation method. In Section 5 we then show how to construct an approximation of the current from the particles that yields a stable scheme, which in the DG case can be seen as a standard deposition method with a local correction. All this is finally validated in Section 6 on two simple but relevant test cases.

\section{Theoretical framework}

To analyze the conforming and non-conforming methods described in the following sections we rely on the tools provided in Section 2 of our companion article [10]. There the 2D Maxwell equations on a bounded domain $\Omega$ were reformulated using a sequence of operators

$$
V^{0} \stackrel{d^{0}=\iota}{\longrightarrow} V^{1} \stackrel{d^{1}=\text { curl }}{\longrightarrow} V^{2} \stackrel{d^{2}=\text { div }}{\longrightarrow} V^{3} \stackrel{0}{\longrightarrow}\{0\}
$$

with $\iota$ the canonical injection from $\mathbb{R}$ to $L^{2}(\Omega)$, and respective domains $V^{0}=\mathbb{R}, V^{1}=H(\operatorname{curl}, \Omega)$, $V^{2}=\boldsymbol{H}(\operatorname{div}, \Omega)$ and $V^{3}=L^{2}(\Omega)$. If $\Omega$ is a bounded and simply-connected Lipschitz domain then the sequence (2.1) is exact, and so is the dual sequence of adjoint operators

$$
\{0\} \stackrel{0}{\longrightarrow} V_{3}^{*} \stackrel{\left(d^{2}\right)^{*}=- \text { grad }}{\longrightarrow} V_{2}^{*} \stackrel{\left(d^{1}\right)^{*}=\text { curl }}{\longrightarrow} V_{1}^{*} \stackrel{\left(d^{1}\right)^{*}=\text { curl }}{\longrightarrow} V_{0}^{*}
$$




\section{Compatible Maxwell solvers With Particles, II}

with domains $V_{3}^{*}=H_{0}^{1}(\Omega), V_{2}^{*}=\boldsymbol{H}_{0}(\operatorname{curl}, \Omega), V_{1}^{*}=L^{2}(\Omega)$ and $V_{0}^{*}=\mathbb{R}$. Letting then $\mathcal{A}$ be defined by

$$
\mathcal{A}=c\left(\begin{array}{cc}
0 & -\left(d^{1}\right)^{*} \\
d^{1} & 0
\end{array}\right)=c\left(\begin{array}{cc}
0 & -\operatorname{curl} \\
\text { curl } & 0
\end{array}\right) \quad \text { on } \quad \mathcal{V}=V^{1} \times V_{2}^{*}=H(\operatorname{curl}, \Omega) \times \boldsymbol{H}_{0}(\operatorname{curl}, \Omega),
$$

the time-dependent Maxwell equations (1.1) with metallic boundary conditions can be rewritten as

$$
\partial_{t} U-\mathcal{A} U=-F
$$

with $U=(c B, \mathbf{E})^{T}, F=\left(0, \varepsilon_{0}^{-1} \boldsymbol{J}\right)^{T}$, and the Gauss laws in the reduced 2D setting can be recast as

$$
\mathcal{D} U=R
$$

where $R:=\left(f_{\Omega} c B^{0}, \varepsilon_{0}^{-1} \rho\right)^{T}$ represents the charge density in this $2 \mathrm{D}$ model, and $\mathcal{D}$ is a composite divergence operator defined by

$$
\mathcal{D}=\left(\begin{array}{cc}
\left(d^{0}\right)^{*} & 0 \\
0 & d^{2}
\end{array}\right)=\left(\begin{array}{cc}
f_{\Omega} & 0 \\
0 & \operatorname{div}
\end{array}\right) \quad \text { on } \quad V_{1}^{*} \times V^{2}=L^{2}(\Omega) \times \boldsymbol{H}(\operatorname{div}, \Omega) .
$$

For completeness we recall the following definitions from Section 2 of [10], as they are central in our stability and error analysis. First a notion of Gauss-compatible approximation was derived from our previous work [11], which allows for long-stable schemes in the case of exact sources (see e.g. Corollaries 2.6 and 2.7 in [10]).

Definition 2.1 (Def. 2.3 from [10]). We say that a discrete operator $\mathcal{A}_{h}: \mathcal{V}_{h} \rightarrow \mathcal{V}_{h}$ forms a Gausscompatible approximation of $\mathcal{A}$ together with a mapping $\Pi_{h}$ on $\mathcal{V}_{h}$ if there exists an auxiliary mapping $\hat{\Pi}_{h}: \hat{\mathcal{V}} \rightarrow \mathcal{V}_{h}$ that converges pointwise to the identity as $h \rightarrow 0$, and that is such that

$$
\Pi_{h} \mathcal{A}=\mathcal{A}_{h} \hat{\Pi}_{h}
$$

holds on $\hat{\mathcal{V}}$.

Then, notions of structure-preserving and charge-conserving discretizations were introduced to guarantee long-time stability estimates in the case of approximate sources as detailed in Sections 2.1 and 2.4 of [10], see in particular Theorem 2.19. Since in this article we design schemes based on the second sequence (2.2), the appropriate definition is as follows.

Definition 2.2 (Def. 2.10 and Lemma 2.13 from [10]). We say that a semi-discrete 2D Maxwell system of the form

$$
\left\{\begin{array} { l } 
{ \partial _ { t } B _ { h } + \operatorname { c u r l } _ { h } \boldsymbol { E } _ { h } = 0 } \\
{ \partial _ { t } \boldsymbol { E } _ { h } - c ^ { 2 } \operatorname { c u r l } _ { h } B _ { h } = - \frac { 1 } { \varepsilon _ { 0 } } \boldsymbol { J } _ { h } }
\end{array} \quad \text { with } \quad \left\{\begin{array}{l}
\operatorname{curl}_{h}: V_{h}^{1} \rightarrow V_{h}^{2} \\
\operatorname{curl}_{h}:=\left(\operatorname{curl}_{h}\right)^{*}: V_{h}^{2} \rightarrow V_{h}^{1}
\end{array}\right.\right.
$$

completed with discrete Gauss laws of the form

$$
\left\{\begin{array} { l } 
{ \operatorname { d i v } _ { h } \boldsymbol { E } _ { h } = \frac { 1 } { \varepsilon _ { 0 } } \rho _ { h } } \\
{ ( \iota _ { h } ) ^ { * } B _ { h } = ( \iota _ { h } ) ^ { * } B _ { h } ^ { 0 } }
\end{array} \quad \text { with } \quad \left\{\begin{array}{l}
\operatorname{div}_{h}: V_{h}^{2} \rightarrow V_{h}^{3} \\
\iota_{h}: V_{h}^{0} \rightarrow V_{h}^{1}
\end{array}\right.\right.
$$

is structure-preserving if the following properties hold.

- Exact sequence property: with $\operatorname{grad}_{h}:=-\left(\operatorname{div}_{h}\right)^{*}$, the sequence

$$
V_{h}^{3} \stackrel{\operatorname{grad}_{h}}{\longrightarrow} V_{h}^{2} \stackrel{\operatorname{curl}_{h}}{\longrightarrow} V_{h}^{1} \stackrel{\left(\iota_{h}\right)^{*}}{\longrightarrow} V_{h}^{0}
$$

is exact, in the sense that $\operatorname{grad}_{h} V_{h}^{3}=\operatorname{ker} \operatorname{curl}_{h}$ and $\operatorname{curl}_{h} V_{h}^{2}=\operatorname{ker}\left(\iota_{h}\right)^{*}$. 


\section{Campos Pinto \& E. Sonnendrǘker}

- Stability: the operators in the above sequence satisfy Poincaré estimates,

$$
\begin{aligned}
\|u\| & \leq c_{P}\left\|\operatorname{grad}_{h} u\right\|, & & u \in V_{h}^{3} \cap(\text { ker grad })^{\perp} \\
\|\boldsymbol{u}\| & \leq c_{P}\left\|\operatorname{curl}_{h} \boldsymbol{u}\right\|, & & \boldsymbol{u} \in V_{h}^{2} \cap\left(\operatorname{ker} \operatorname{curl}_{h}\right)^{\perp} \\
\|u\| & \leq c_{P}\left\|\left(\iota_{h}\right)^{*} u\right\|, & & u \in V_{h}^{1} \cap\left(\operatorname{ker}\left(\iota_{h}\right)^{*}\right)^{\perp} .
\end{aligned}
$$

with a constant $c_{P}$ independent of $h$.

In Lemma 2.14 from [10] it is observed that if the discrete system (2.6) is put under the form

$$
\left\{\begin{aligned}
\partial_{t} U_{h}-\mathcal{A}_{h} U_{h} & =-F_{h} \\
\mathcal{D}_{h} U_{h} & =R_{h}
\end{aligned}\right.
$$

with

$\mathcal{A}_{h}:=c\left(\begin{array}{cc}0 & -\operatorname{curl}_{h} \\ \operatorname{curl}_{h} & 0\end{array}\right):\left(V_{h}^{1} \times V_{h}^{2}\right) \rightarrow\left(V_{h}^{1} \times V_{h}^{2}\right), \quad \mathcal{D}_{h}:=\left(\begin{array}{cc}\left(\iota_{h}\right)^{*} & 0 \\ 0 & \operatorname{div}_{h}\end{array}\right):\left(V_{h}^{1} \times V_{h}^{2}\right) \rightarrow\left(V_{h}^{0} \times V_{h}^{3}\right)$ then properties (2.8)-(2.9) hold if and only if the composite curl and divergence operators satisfy:

$$
\begin{array}{lll}
\text { (i) } & \|Z\| \leq c_{P}\left\|\mathcal{A}_{h} Z\right\|, \quad Z \in\left(\operatorname{ker} \mathcal{A}_{h}\right)^{\perp} & \text { (unif. stability of } \left.\mathcal{A}_{h}\right) \\
\text { (ii) } & \|Z\| \leq c_{P}\left\|\mathcal{D}_{h} Z\right\|, \quad Z \in\left(\operatorname{ker} \mathcal{D}_{h}\right)^{\perp} & \text { (unif. stability of } \left.\mathcal{D}_{h}\right) \\
\text { (iii) } & \operatorname{ker} \mathcal{D}_{h}=\left(\operatorname{ker} \mathcal{A}_{h}\right)^{\perp} & \text { (compatibility of the kernels). }
\end{array}
$$

The purpose of Definition 2.2 is to guarantee the long-time stability of the solutions to the full discrete Maxwell system (2.10). A criterion on the discrete sources is then introduced to guarantee that solutions to the discrete Ampère and Faraday equations also satisfy the proper discrete Gauss law.

Definition 2.3 (Def. 2.15 from [10]). We say that a semi-discrete Maxwell system of the form (2.10) with $\mathcal{A}_{h}$ a skew-symmetric operator, is charge-conserving if

(i) it is structure-preserving in the sense that properties (2.11), (2.12) and (2.13) hold,

(ii) and the approximate sources satisfy the corresponding discrete continuity equation

$$
\partial_{t} R_{h}+\mathcal{D}_{h} F_{h}=0 .
$$

Finally we observe that with the notation of Definition 2.2, the first equation from (2.14) is trivial and the second one rewrites as

$$
\partial_{t} \rho_{h}+\operatorname{div}_{h} \boldsymbol{J}_{h}=0 .
$$

\section{Conforming elements for the 2D Maxwell system with a strong Faraday law}

Although it makes no difference on the continuous problem whether one takes the sequence (2.1) or its dual version (2.2) for the primal complex, on the discrete level it leads to two different types of Galerkin methods. In our companion article [10] we have described the first choice which leads to a strong discretization of the Ampère equation with natural boundary conditions. In this article we consider the second choice which leads to a strong discrete Faraday equation with essential boundary conditions.

Because the construction of our new non-conforming method relies on a good understanding of the conforming tools, we now verify that the structure of the Finite Element exterior calculus introduced by Arnold, Falk and Winther [1, 2, 3], linking the conforming Galerkin approximations of the different Hilbert spaces perfectly fits in the framework introduced in the previous sections. Specifically, this will allow us to show in Section 3.4 that a standard sequence of Finite Element spaces can be equipped 


\section{Compatible Maxwell solvers With Particles, II}

with a Gauss-compatible approximation operator in the sense of Definition 2.1, and in Section 5.1 we will verify that it naturally yields a structure-preserving discretization of Maxwell's equations in the sense of Definition 2.2. Our non-conforming approximation will consist in giving more freedom in the choice of the discrete spaces, in particular to include discontinuous broken spaces, by carefully choosing the projection operators and discrete differential operators so as to preserve the compatibility and structure-preserving properties.

\subsection{Mesh notations}

For the mesh elements we use the same notations as in [10]. In particular, we assume that the domain $\Omega$ is partitioned by a regular family of conforming simplicial meshes $\mathcal{T}_{h}$ with maximal triangle diameter $h$ tending to zero. We denote by $\mathcal{E}(T)$ the edges of a triangle $T \in \mathcal{T}_{h}$, and $\mathcal{E}_{h}:=\cup_{T \in \mathcal{T}_{h}} \mathcal{E}(T)$ the set of all the edges in the mesh. Boundary edges are stored in $\mathcal{E}_{h}^{B}$. Assuming that the triangles in $\mathcal{T}_{h}$ are given arbitrary indices $i=0, \ldots, \#\left(\mathcal{T}_{h}\right)-1$, we fix an orientation for the edges as follows. Given $e \in \mathcal{E}_{h}$, we let $T^{-}(e)$ be the triangle of minimum index for which $e$ is an edge, and if $e$ is not a boundary edge we denote by $T^{+}(e)$ the other triangle sharing $e$. The edge $e$ is then oriented by setting

$$
\boldsymbol{n}_{e}:=\boldsymbol{n}_{e}^{T^{-}(e)},
$$

where $\boldsymbol{n}_{e}^{T}$ denotes the outward unit vector of $T$ that is normal to $e$, for any $e \in \mathcal{E}(T)$.

\subsection{Conforming Finite Elements with a strong Faraday law}

To derive finite element schemes with a strong Faraday equation we approximate the non-trivial spaces in the dual sequence (2.2), i.e.,

$$
V_{3}^{*}=H_{0}^{1}(\Omega) \stackrel{\left(d^{2}\right)^{*}=- \text { grad }}{\longrightarrow} V_{2}^{*}=\boldsymbol{H}_{0}(\operatorname{curl} ; \Omega) \stackrel{\left(d^{1}\right)^{*}=\text { curl }}{\longrightarrow} V_{1}^{*}=L^{2}(\Omega)
$$

by a sequence of discrete spaces $V_{h}^{3} \stackrel{\left(d_{h}^{2}\right)^{*}}{\longrightarrow} V_{h}^{2} \stackrel{\left(d_{h}^{1}\right)^{*}}{\longrightarrow} V_{h}^{1}$. In this section we opt for a conforming sequence, i.e., such that

$$
V_{h}^{3} \subset H_{0}^{1}(\Omega), \quad V_{h}^{2} \subset \boldsymbol{H}_{0}(\operatorname{curl} ; \Omega), \quad V_{h}^{1} \subset L^{2}(\Omega),
$$

so that $\left(d_{h}^{l}\right)^{*}$ can be defined as the restriction of $\left(d^{l}\right)^{*}$ to $V_{h}^{l+1}$. Observe that here we denote standard (strong) differential operators as dual ones, and conversely the plain notation $d_{h}^{l}: V_{h}^{l} \rightarrow V_{h}^{l+1}$ will be used to denote the discrete adjoint of $\left(d_{h}^{l}\right)^{*}$, which only makes sense in a weak form, using test functions from $V_{h}^{l+1}$. This rather unusual choice is motivated by our desire to use notations consistent with our companion article [10] where the discretization is performed on the sequence (2.1) which is seen as the primal one, but obviously the other choice could be made as well by calling (2.2) the primal sequence.

Again, several options are possible for the conforming spaces in (3.2). Here we focus on a standard strategy (see, e.g., [20]) where the spaces $V_{h}^{l}$ are respectively defined as a continuous Finite Element space, a Nédélec Finite Element space of the first kind and a discontinuous Galerkin Finite Element space, but we note that other choices are possible, see e.g. Remark 4.7 below. Specifically, given an integer degree $p \geq 1$ we take

$$
V_{h}^{3}:=\mathcal{L}_{p, 0}\left(\Omega, \mathcal{T}_{h}\right) \stackrel{\left(d_{h}^{2}\right)^{*}:=-\left.\operatorname{grad}\right|_{V_{h}^{3}}}{\longrightarrow} V_{h}^{2}:=\mathcal{N}_{p-1,0}\left(\Omega, \mathcal{T}_{h}\right) \stackrel{\left(d_{h}^{1}\right)^{*}:=\left.\operatorname{curl}\right|_{V_{h}^{2}}}{\longrightarrow} V_{h}^{1}:=\mathbb{P}_{p-1}\left(\mathcal{T}_{h}\right)
$$

where

$$
\mathbb{P}_{p-1}\left(\mathcal{T}_{h}\right):=\left\{v \in L^{2}(\Omega):\left.v\right|_{T} \in \mathbb{P}_{p-1}(T), T \in \mathcal{T}_{h}\right\}
$$




\section{Campos Pinto \& E. Sonnendrǘcker}

denotes the space of piecewise polynomials of maximal degree $p-1$ on the triangulation $\mathcal{T}_{h}$,

$$
\mathcal{L}_{p, 0}\left(\Omega, \mathcal{T}_{h}\right):=\mathbb{P}_{p}\left(\mathcal{T}_{h}\right) \cap \mathcal{C}_{0}(\Omega)
$$

corresponds to the continuous "Lagrange" elements with homogeneous boundary conditions, and

$$
\mathcal{N}_{p-1,0}\left(\Omega, \mathcal{T}_{h}\right):=\mathcal{N}_{p-1}\left(\mathcal{T}_{h}\right) \cap \boldsymbol{H}_{0}(\operatorname{curl} ; \Omega) \quad \text { with } \quad \mathcal{N}_{p-1}(T):=\mathbb{P}_{p-1}(T)^{2}+\left(\begin{array}{c}
-y \\
x
\end{array}\right) \mathbb{P}_{p-1}(T)
$$

is the (first-kind) Nédélec Finite Element space of order $p-1$ (thus of maximal degree $p$ ), again with homogeneous boundary conditions, see e.g., [4].

Remark 3.1. To be conforming in $\boldsymbol{H}(\operatorname{curl} ; \Omega)$, the piecewise polynomial space $V_{h}^{2}$ must be composed of vector fields that have no tangential discontinuities on the edges of the mesh, in the sense that for any $\boldsymbol{u} \in V_{h}^{2}$ the tangential trace $\boldsymbol{n}_{e} \times \boldsymbol{u}$ on an edge $e$ must be the same when defined either from $T^{-}(e)$ or from $T^{+}(e)$. In particular, every basis of $V_{h}^{2}$ must contain some vector fields supported on two adjacent cells at least.

For the sake of completeness we recall the following well-kown result which will be central to our analysis. The proof is almost the same as the one of Lemma 3.2 in [10], up to the boundary conditions which are treated by straightforward considerations, and will be skipped.

Lemma 3.2. The following sequence is exact, in the sense that the range of each operator coincides with the kernel of the following operator,

$$
\{0\} \stackrel{0}{\longrightarrow} V_{h}^{3}=\mathcal{L}_{p, 0}\left(\Omega, \mathcal{T}_{h}\right) \stackrel{\left(d_{h}^{2}\right)^{*}=-\operatorname{grad}}{\longrightarrow} V_{h}^{2}=\mathcal{N}_{p-1,0}\left(\Omega, \mathcal{T}_{h}\right) \stackrel{\left(d_{h}^{1}\right)^{*}=\text { curl }}{\longrightarrow} V_{h}^{1}=\mathbb{P}_{p-1}\left(\mathcal{T}_{h}\right) \stackrel{f_{\Omega}}{\longrightarrow} \mathbb{R}
$$

where we remind that $f_{\Omega}: u \mapsto|\Omega|^{-1} \int_{\Omega} u$.

Based on the above spaces, a standard conforming Finite Element method consists in computing the unique solution $\left(B_{h}, \boldsymbol{E}_{h}\right) \in \mathcal{C}^{0}\left([0, T] ; V_{h}^{1} \times V_{h}^{2}\right)$ to

$$
\left\{\begin{aligned}
\left\langle\partial_{t} B_{h}, \varphi\right\rangle+\left\langle\operatorname{curl} \boldsymbol{E}_{h}, \varphi\right\rangle & =0 & & \varphi \in V_{h}^{1} \subset L^{2}(\Omega) \\
\left\langle\partial_{t} \boldsymbol{E}_{h}, \boldsymbol{\varphi}\right\rangle-c^{2}\left\langle B_{h}, \operatorname{curl} \boldsymbol{\varphi}\right\rangle & =-\frac{1}{\varepsilon_{0}}\left\langle\boldsymbol{J}_{h}, \boldsymbol{\varphi}\right\rangle & & \boldsymbol{\varphi} \in V_{h}^{2} \subset \boldsymbol{H}_{0}(\operatorname{curl} ; \Omega)
\end{aligned}\right.
$$

where $\boldsymbol{J}_{h} \in \mathcal{C}^{0}\left([0, T] ; V_{h}^{2}\right)$ represents an approximation of the given current density $\boldsymbol{J}$ and $\langle\cdot, \cdot\rangle$ stands for the scalar product in $L^{2}(\Omega)$. Note that using the embedding curl $V_{h}^{2} \subset V_{h}^{1}$ the second equation amounts to

$$
\partial_{t} B_{h}+\operatorname{curl} \boldsymbol{E}_{h}=0 \quad\left(\text { in } V_{h}^{1}\right)
$$

which justifies our "strong Faraday" terminology.

This space discretization is standard ans has been studied in, e.g., Ref. [20, 19, 25, 9] where the source term $\boldsymbol{J}$ is approximated with a standard orthogonal projection on $V_{h}^{2}$, leading to define $\boldsymbol{J}_{h}$ by $\left\langle\boldsymbol{J}_{h}, \boldsymbol{\varphi}\right\rangle=\langle\boldsymbol{J}, \boldsymbol{\varphi}\rangle$ for $\boldsymbol{\varphi} \in V_{h}^{2}$. We will see that this approximation of the source term gives a compatible scheme. In the non-conforming case however, we will need to use a different approximation operator, see Theorem 4.2.

\subsection{Projection operators and commuting diagram properties}

In the conforming Finite Element case the projection operators and commuting diagram properties have been discussed and described in a series of papers by Arnold, Falk and Winther on what they call the Finite Element Exterior Calculus (FEEC) [1, 2,3]. For the present paper we shall only need 


\section{Compatible Maxwell solvers With Particles, II}

to review the properties of $\pi_{h}^{\text {curl }}$, the canonical projection on the curl-conforming Nédélec space. In 2D this projection uses edge and face based degrees of freedom, and it satisfies a commuting diagram

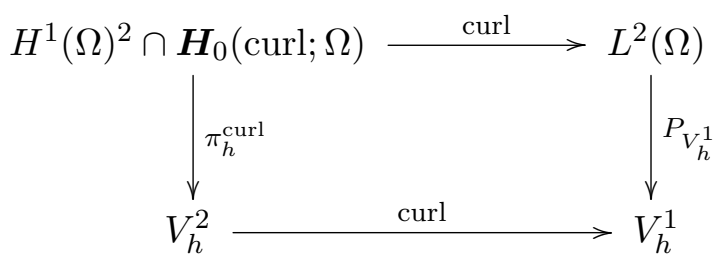

involving the orthogonal projection on the discontinuous space $V_{h}^{1}$, see Equation (3.12) below.

As a matter of fact, the canonical projection on the Nédélec space can be derived from the standard Raviart-Thomas interpolation (recalled in our companion article [10]) by a rotation of $\pi / 2$. Specifically, the degrees of freedom for the finite element space $V_{h}^{2}=\mathcal{N}_{p-1,0}\left(\Omega, \mathcal{T}_{h}\right)$ read (see, e.g., [21] or [15])

$$
\begin{cases}\mathcal{M}_{h}^{2}(T, \boldsymbol{u}):=\left\{\int_{T} \boldsymbol{u} \cdot \boldsymbol{q}: \boldsymbol{q} \in \mathbb{P}_{p-2}(T)^{2}\right\} & \text { for every triangle } T \in \mathcal{T}_{h}, \\ \mathcal{M}_{h}^{2}(e, \boldsymbol{u}):=\left\{\int_{e}\left(\boldsymbol{n}_{e} \times \boldsymbol{u}\right) q: q \in \mathbb{P}_{p-1}(e)\right\} & \text { for every edge } e \in \mathcal{E}_{h} \backslash \mathcal{E}_{h}^{B}\end{cases}
$$

where $\mathcal{E}_{h}^{B}$ denotes the set of boundary edges. The Nédélec finite element interpolation

$$
\pi_{h}^{\text {curl }}: H^{1}(\Omega)^{2} \rightarrow V_{h}^{2}:=\mathcal{N}_{p-1,0}\left(\Omega, \mathcal{T}_{h}\right)
$$

is then defined by the relations

$$
\mathcal{M}_{h}^{2}\left(T, \pi_{h}^{\text {curl }} \boldsymbol{u}-\boldsymbol{u}\right)=\{0\}, \quad T \in \mathcal{T}_{h} \quad \text { and } \quad \mathcal{M}_{h}^{2}\left(e, \pi_{h}^{\text {curl }} \boldsymbol{u}-\boldsymbol{u}\right)=\{0\}, \quad e \in \mathcal{E}_{h} \backslash \mathcal{E}_{h}^{B} .
$$

Again, this interpolation satisfies a commuting diagram property which is easily verified using integration by parts,

$$
\operatorname{curl} \pi_{h}^{\text {curl }} \boldsymbol{u}=P_{V_{h}^{1}} \operatorname{curl} \boldsymbol{u}, \quad \boldsymbol{u} \in H^{1}(\Omega)^{2} \cap \boldsymbol{H}_{0}(\operatorname{curl} ; \Omega)
$$

where $P_{V_{h}^{1}}$ denotes the $L^{2}$ projection on the discontinuous space $V_{h}^{1}=\mathbb{P}_{p-1}\left(\mathcal{T}_{h}\right)$, and a classical error estimate

$$
\left\|\pi_{h}^{\text {curl }} \boldsymbol{u}-\boldsymbol{u}\right\| \leq c h^{m}|\boldsymbol{u}|_{m}, \quad 1 \leq m \leq p, \quad \boldsymbol{u} \in \boldsymbol{H}_{0}(\operatorname{curl} ; \Omega) .
$$

Remark 3.3. As is well known, in $2 \mathrm{D}$ the Nédélec finite element space $\mathcal{N}_{p-1}$ can be obtained by rotating the Raviart-Thomas space $\boldsymbol{R}_{p-1}$ by an angle of $\pi / 2$ : we have $\mathcal{N}_{p-1}(T)=R \mathcal{R T}_{p-1}(T)$ where $R: \boldsymbol{u} \mapsto\left(-u_{y}, u_{x}\right)$. Up to the boundary conditions (for which the degrees of freedom need to be added or substracted from the respective bases), it is then easily verified that $\pi_{h}^{\text {curl }}=R \pi_{h}^{\text {div }} R^{-1}$, so that the properties listed here for the Nédélec interpolation can be derived from those of the Raviart-Thomas interpolation recalled in [10], using the identity $\operatorname{curl}=\operatorname{div} R^{-1}$.

When designing non-conforming approximations based on broken spaces in Section 4.1 it will be convenient to follow [13] as we have done in [10] for the non-conforming strong Ampère scheme, and use a basis for $V_{h}^{2}$ that is dual to the above degrees of freedom. Its construction goes as follows. Given scalar-valued bases $q_{e, i}, i=1, \ldots p$ for the edge polynomials $\mathbb{P}_{p-1}(e), e \in \mathcal{E}_{h} \backslash \mathcal{E}_{h}^{B}$, and vector-valued bases $\boldsymbol{q}_{T, i}, i=1, \ldots, p(p-1)$ for the "volume" polynomials $\mathbb{P}_{p-2}(T)^{2}, T \in \mathcal{T}_{h}$, we span the moment spaces listed in (3.10) with the degrees of freedom

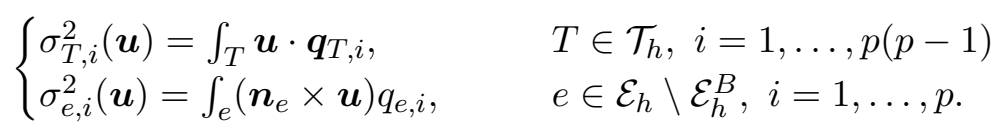

It follows from the unisolvence of (3.10) that $\mathcal{N}_{p-1}(T)$ admits a unique basis $\varphi_{\lambda}^{2, T}$ with indices in

$$
\Lambda^{2}(T):=\Lambda_{\mathrm{vol}}^{2}(T) \cup \Lambda_{\text {edge }}^{2}(T) \quad \text { where } \quad\left\{\begin{array}{l}
\Lambda_{\mathrm{vol}}^{2}(T):=\{(T, i): i=1, \ldots, p(p-1)\} \\
\Lambda_{\text {edge }}^{2}(T):=\{(e, i): e \in \mathcal{E}(T), i=1, \ldots, p\}
\end{array}\right.
$$




\section{Campos Pinto \& E. Sonnendrǘker}

that is dual to the associated degrees of freedom, in the sense that we have $\sigma_{\gamma}^{2}\left(\varphi_{\lambda}^{2, T}\right)=\delta_{\gamma, \lambda}$ for $\gamma, \lambda \in \Lambda^{2}(T)$. To form a basis of the global space $V_{h}^{2}$ we then gather all the indices, except for those attached to boundary edges, into

$$
\Lambda_{h}^{2}:=\left(\cup_{T \in \mathcal{T}_{h}} \Lambda^{2}(T)\right) \backslash\left\{(e, i): e \in \mathcal{E}_{h}^{B}, i=1, \ldots p\right\} .
$$

The curl-conformity of (3.10) then guarantees that if we set $\varphi_{\lambda}^{2, T}:=0$ for $\lambda \in \Lambda_{h}^{2} \backslash \Lambda^{2}(T)$ and if we extend $\varphi_{\lambda}^{2, T}$ by 0 outside $T$ for $\lambda \in \Lambda^{2}(T)$, then the piecewise polynomials

$$
\varphi_{\lambda}^{2}:=\sum_{T \in \mathcal{T}_{h}} \mathbb{1}_{T} \varphi_{\lambda}^{2, T}=\sum_{T \in \mathcal{T}_{h}} \varphi_{\lambda}^{2, T}
$$

are in $\boldsymbol{H}_{0}(\operatorname{curl} ; \Omega)$ and they form a basis for the space $V_{h}^{2}$ that is dual to the associated degrees of freedom in the sense that

$$
\sigma_{\gamma}^{2}\left(\varphi_{\lambda}^{2}\right)=\delta_{\gamma, \lambda} \quad \text { for } \gamma, \lambda \in \Lambda_{h}^{2}
$$

Moreover, if the polynomials $q_{e, i}$ and $\boldsymbol{q}_{T, i}$ involved in (3.14) are defined as suitable affine maps of polynomial bases defined on a reference element, the resulting local basis functions $\varphi_{\lambda}^{2, T}$ will also correspond to affine maps of the associated reference basis. As a result, if the mesh $\mathcal{T}_{h}$ is shape regular in the standard sense of, e.g., [15, Def. I-A.2], it is possible to ask for normalized local basis functions satisfying, e.g.,

$$
\left\|\varphi_{\lambda}^{2, T}\right\| \sim 1 \quad \text { for } T \in \mathcal{T}_{h}, \lambda \in \Lambda^{2}(T) .
$$

\subsection{Gauss-compatibility of the conforming FEM-Faraday scheme}

In compact form, the conforming scheme (3.7) reads $\partial_{t} U_{h}-\mathcal{A}_{h} U_{h}=-F_{h}$ with $U_{h}=\left(c B_{h}, \boldsymbol{E}_{h}\right)^{T}$ and $F_{h}=\left(0, \varepsilon_{0}^{-1} \boldsymbol{J}_{h}\right)^{T}$. The composite curl operator is defined on $\mathcal{V}_{h}:=V_{h}^{1} \times V_{h}^{2}$ by

$$
\mathcal{A}_{h}:=c\left(\begin{array}{cc}
0 & -\operatorname{curl}_{h} \\
\operatorname{curl}_{h} & 0
\end{array}\right) \quad \text { with } \quad\left\{\begin{array}{l}
\operatorname{curl}_{h}:=\left.\operatorname{curl}\right|_{V_{h}^{2}}: V_{h}^{2} \rightarrow V_{h}^{1} \\
\operatorname{curl}_{h}:=\left(\operatorname{curl}_{h}\right)^{*}: V_{h}^{1} \rightarrow V_{h}^{2} .
\end{array}\right.
$$

Following Definition 2.1 and considering source and auxiliary approximation operators of the form

$$
\Pi_{h}=\left(\begin{array}{cc}
\pi_{h}^{1} & 0 \\
0 & \pi_{h}^{2}
\end{array}\right) \quad \text { and } \quad \hat{\Pi}_{h}=\left(\begin{array}{cc}
\hat{\pi}_{h}^{1} & 0 \\
0 & \hat{\pi}_{h}^{2}
\end{array}\right)
$$

we then see that this scheme is Gauss-compatible on some product space $\hat{V}^{1} \times \hat{V}^{2}$ if for $l=1,2$ we can find approximation operators $\pi_{h}^{l}$ and $\hat{\pi}_{h}^{l}$ mapping on $V_{h}^{l}$, such that

$$
\pi_{h}^{2} \operatorname{curl} u=\left(\operatorname{curl}_{h}\right)^{*} \hat{\pi}_{h}^{1} u, \quad u \in \hat{V}^{1}
$$

and

$$
\pi_{h}^{1} \operatorname{curl} \boldsymbol{u}=\operatorname{curl} \hat{\pi}_{h}^{2} \boldsymbol{u}, \quad \boldsymbol{u} \in \hat{V}^{2} .
$$

Note that these relations read $\pi_{h}^{2} d^{1} u=d_{h}^{1} \hat{\pi}_{h}^{1} u$ and $\pi_{h}^{1}\left(d^{1}\right)^{*} \boldsymbol{u}=\left(d_{h}^{1}\right)^{*} \hat{\pi}_{h}^{2} \boldsymbol{u}$ with the notations of Section 2 and 3.2 .

Theorem 3.4. The conforming FEM-Faraday scheme (3.7) associated with an orthogonal projection for the current, namely

$$
\pi_{h}^{2}:=P_{V_{h}^{2}}: L^{2}(\Omega)^{2} \rightarrow V_{h}^{2}=\mathcal{N}_{p-1,0}\left(\Omega, \mathcal{T}_{h}\right),
$$

see (3.6), is Gauss-compatible on the product space

$$
\hat{V}^{1} \times \hat{V}^{2}:=V^{1} \times\left(H^{1}(\Omega) \cap V_{2}^{*}\right)
$$




\section{Compatible Maxwell solvers With Particles, II}

where $V^{1}=H(\operatorname{curl} ; \Omega)$ and $V_{2}^{*}=\boldsymbol{H}_{0}(\operatorname{curl} ; \Omega)$, see Section 2. In particular, Equation (3.19) holds with an $L^{2}$-projection $\hat{\pi}_{h}^{1}:=P_{V_{h}^{1}}$ and Equation (3.20) holds with $\pi_{h}^{1}:=P_{V_{h}^{1}}$ and $\hat{\pi}_{h}^{2}:=\pi_{h}^{\text {curl }}$ the canonical (Nédélec) interpolation on $V_{h}^{2}$ defined in (3.11). Moreover, these mappings satisfy

$$
\begin{array}{rlrl}
\left\|\hat{\pi}_{h}^{1} u-u\right\| & \lesssim h^{m}|u|_{m}, & & 0 \leq m \leq p \\
\left\|\hat{\pi}_{h}^{2} \boldsymbol{u}-\boldsymbol{u}\right\| \lesssim h^{m}|\boldsymbol{u}|_{m}, & & 1 \leq m \leq p, \quad \boldsymbol{u} \in \boldsymbol{H}_{0}(\operatorname{curl} ; \Omega) \\
\left\|\pi_{h}^{1} u-u\right\| \lesssim h^{m}|u|_{m}, & & 0 \leq m \leq p \\
\left\|\pi_{h}^{2} \boldsymbol{u}-\boldsymbol{u}\right\| \lesssim h^{m}|\boldsymbol{u}|_{m}, & & 0 \leq m \leq p .
\end{array}
$$

Proof. Let us first show that the relation (3.19) holds as claimed, that is,

$$
P_{V_{h}^{2}} d^{1} u=d_{h}^{1} P_{V_{h}^{1}} u, \quad u \in V^{1} .
$$

Since both sides belong to $V_{h}^{2}$ by construction, one can test this equality against an arbitrary $\boldsymbol{v} \in V_{h}^{2}$, which is in $V_{2}^{*}$ by conformity (3.2). Using the definition of the various operators, in particular the fact that $\left(d_{h}^{1}\right)^{*}$ is defined as the restriction of $\left(d^{1}\right)^{*}$ to $V_{h}^{2}$, we compute

$$
\left\langle P_{V_{h}^{2}} d^{1} u, \boldsymbol{v}\right\rangle=\left\langle d^{1} u, \boldsymbol{v}\right\rangle=\left\langle u,\left(d^{1}\right)^{*} \boldsymbol{v}\right\rangle=\left\langle u,\left(d_{h}^{1}\right)^{*} \boldsymbol{v}\right\rangle=\left\langle P_{V_{h}^{1}} u,\left(d_{h}^{1}\right)^{*} \boldsymbol{v}\right\rangle=\left\langle d_{h}^{1} P_{V_{h}^{1}} u, \boldsymbol{v}\right\rangle,
$$

which proves (3.19). On the other hand, (3.20) is nothing but the commuting diagram (3.12). Finally the error estimates are standard for $L^{2}$ projections, and (3.22) is (3.13).

If one is solving the Maxwell equations with exact sources, Theorem 2.5 from [10] applies and gives the following a priori estimate.

Corollary 3.5. Let $(B, \boldsymbol{E})$ be the exact solution to the Maxwell system (2.3). The semi-discrete solution to the FEM-Faraday scheme (3.7) coupled with the orthogonal projection (3.21) for the current satisfies

$$
\begin{aligned}
\left\|\left(B-B_{h}\right)(t)\right\|+\left\|\left(\boldsymbol{E}-\boldsymbol{E}_{h}\right)(t)\right\| & \lesssim\left\|B_{h}(0)-\hat{\pi}_{h}^{1} B(0)\right\|+\left\|\boldsymbol{E}_{h}(0)-\hat{\pi}_{h}^{2} \boldsymbol{E}(0)\right\| \\
& +h^{m}\left(|B(0)|_{m}+\int_{0}^{t}\left|\partial_{t} B(s)\right|_{m} \mathrm{~d} s\right)+h^{m^{\prime}}\left(|\boldsymbol{E}(0)|_{m^{\prime}}+\int_{0}^{t}\left|\partial_{t} \boldsymbol{E}(s)\right|_{m^{\prime}} \mathrm{d} s\right)
\end{aligned}
$$

for $0 \leq m \leq p, 1 \leq m^{\prime} \leq p$, and with a constant independent of $h$ and $t$.

Remark 3.6. A priori estimates leading to long-time stability are known already for the strong Faraday scheme $(3.7)$, see $[20,19,25]$. The main benefit of our analysis is that it readily applies to Maxwell solvers for which the $L^{2}$ projection is not a compatible approximation operator, and nonconforming discretizations, see e.g. Section 4.2.

Remark 3.7. In the case of approximate sources, one must resort to the analysis developped in Section 2.4 of [10] to be able to derive long-time stability estimates. This will be done in Section 5.1 by showing that the FEM-Faraday scheme (3.7) is naturally structure-preserving.

\section{Discontinuous elements for the 2D Maxwell system with a strong Faraday law}

Because of its weak formulation, discretizing in time the Ampère law from (3.7) requires to invert a mass matrix associated with the space $V_{h}^{2}$, and due to the curl-conformity of the latter space the resulting inversion can not be performed locally. This can of course become a computational burden when the meshes become very fine and when parallel algorithms come into play. For this reason we study a non-conforming method where the solution is approximated in a fully discontinuous space,

$$
\tilde{V}_{h}^{2} \not \subset V_{2}^{*}=\boldsymbol{H}_{0}(\operatorname{curl} ; \Omega) \text {. }
$$




\section{Campos Pinto \& E. Sonnendrǘker}

It turns out from our analysis that a natural choice corresponds to using the broken Nédélec space

$$
\tilde{V}_{h}^{2}:=\mathcal{N}_{p-1}\left(\mathcal{T}_{h}\right)=\left\{\boldsymbol{u} \in L^{2}(\Omega):\left.\boldsymbol{u}\right|_{T} \in \mathcal{N}_{p-1}(T), T \in \mathcal{T}_{h}\right\}
$$

see (3.6). Standard polynomial spaces are also a possible choice, as discussed in Remark 4.7 below.

Although the common way for designing non-conforming discretizations is to follow the discontinuous Galerkin methodology (see e.g. $[14,18]$ ), in this work we aim at preserving a strong Faraday equation like (3.8). For that purpose we experiment a different path and apply to the 2D problem the ideas of the Conga discretization proposed and studied in [11] for the 3D Maxwell system (the name standing for "Conforming/Non-conforming Galerkin"). In particular, our non-conforming discretization will be derived from the conforming one (3.3). As we did for the conforming case, and following our 3D study [11], we will show that this non-conforming discretization can be equipped with a Gauss-compatible approximation operator in the sense of Definition 2.1. In Section 5.2 we will extend this analysis by further verifying that it is essentially a structure-preserving discretization of Maxwell's equations in the sense of Definition 2.2, once associated with a nonstandard discrete divergence. Interestingly, we will see that in the $2 \mathrm{D}$ setting the resulting method can be interpreted as a centered DG method, see Section 4.3.

\subsection{Non-conforming "Conga" discretization}

To extend the conforming method (3.7) on the non-conforming space $\tilde{V}_{h}^{2}$ we consider a smoothing operator

$$
\mathcal{P}_{h}^{2}: L^{2}(\Omega)^{2} \rightarrow V_{h}^{2}
$$

(which is not required to satisfy a commuting diagram) and we define the associated Conga approximation $\left(B_{h}, \boldsymbol{E}_{h}\right) \in \mathcal{C}^{0}\left([0, T] ; V_{h}^{1} \times \tilde{V}_{h}^{2}\right)$ by the system

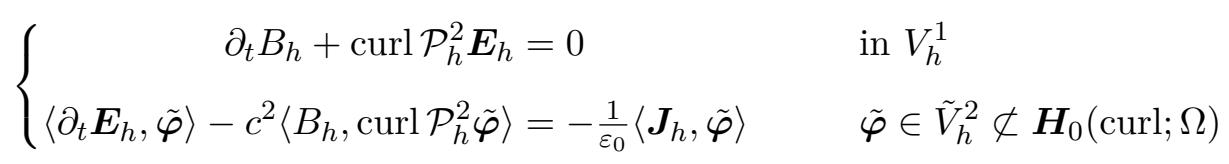

where again $\boldsymbol{J}_{h}$ represents an appropriate approximation (in $\tilde{V}_{h}^{2}$ ) of the current density $\boldsymbol{J}$. Below we will see that Gauss-compatible and charge-conserving schemes cannot be obtained with a straightforward $L^{2}$ projection as in the conforming case.

For the smoothing projection $\mathcal{P}_{h}^{2}$ one may think of using the $L^{2}$ projection on the conforming space $V_{h}^{2}$, but this would have the downside of requiring to invert a $V_{h}^{2}$ mass matrix, a global computation that we precisely whish to avoid. Similarly as in $[11,10]$ we thus use an averaging procedure based on the canonical degrees of freedom for the curl-conforming space $V_{h}^{2}$, here (3.10). To obtain a stable projection in $L^{2}$ we can recycle the elegant construction proposed in [13], where on each mesh triangle $T$ the authors use the local basis that is dual to the broken basis built in Section 3.3, namely the basis $\boldsymbol{\psi}_{\lambda}^{2, T}, \lambda \in \Lambda^{2}(T)$, of $\boldsymbol{\mathcal { N }}_{p-1}(T)$ that is defined by the relations

$$
\left\langle\boldsymbol{\psi}_{\lambda}^{2, T}, \boldsymbol{\varphi}_{\gamma}^{2, T}\right\rangle=\sigma_{\lambda}^{2}\left(\left.\varphi_{\gamma}^{2, T}\right|_{T}\right)=\delta_{\lambda, \gamma} \quad \text { for } \quad \lambda, \gamma \in \Lambda^{2}(T) .
$$

A convenient projection operator $\mathcal{P}_{h}^{2}: L^{2}(\Omega)^{2} \rightarrow V_{h}^{2}$ is then given by

$$
\mathcal{P}_{h}^{2} \boldsymbol{u}:=\sum_{\lambda \in \Lambda_{h}^{2}} \sum_{T \in \mathcal{T}_{h}(\lambda)} \frac{\left\langle\boldsymbol{u}, \boldsymbol{\psi}_{\lambda}^{2, T}\right\rangle}{\#\left(\mathcal{T}_{h}(\lambda)\right)} \boldsymbol{\varphi}_{\lambda}^{2}
$$

where $\mathcal{T}_{h}(\lambda):=\left\{T \in \mathcal{T}_{h}: \lambda \in \Lambda^{2}(T)\right\}$ denotes the cells for which $\lambda$ is an active index. Using (3.15) and (4.4) we verify easily that this is indeed a projection on $V_{h}^{2}=\operatorname{Span}\left(\left\{\varphi_{\lambda}^{2}: \lambda \in \Lambda_{h}^{2}\right\}\right)$. More 


\section{Compatible Maxwell solvers With Particles, II}

precisely, on the broken Nédélec space it amounts to averaging the broken version of the degrees of freedom (3.14):

$$
\boldsymbol{u} \in \mathcal{N}_{p-1}\left(\mathcal{T}_{h}\right) \Longrightarrow \begin{cases}\sigma_{T, i}^{2}\left(\mathcal{P}_{h}^{2} \boldsymbol{u}\right)=\sigma_{T, i}^{2}\left(\left.\boldsymbol{u}\right|_{T}\right)=\sigma_{T, i}^{2}(\boldsymbol{u}), & T \in \mathcal{T}_{h}, i=1, \ldots, p(p-1) \\ \sigma_{e, i}^{2}\left(\mathcal{P}_{h}^{2} \boldsymbol{u}\right)=\frac{1}{2}\left(\sigma_{e, i}^{2}\left(\left.\boldsymbol{u}\right|_{T^{-}(e)}\right)+\sigma_{e, i}^{2}\left(\left.\boldsymbol{u}\right|_{T^{+}(e)}\right)\right), & e \in \mathcal{E}_{h} \backslash \mathcal{E}_{h}^{B}, i=1, \ldots, p\end{cases}
$$

where we remind that $T^{ \pm}(e)$ are the two triangles that share the interior edge $e$. Indeed, decomposing $\boldsymbol{u}=\sum_{T \in \mathcal{T}_{h}, \lambda \in \Lambda^{2}(T)} c_{\lambda}^{T} \boldsymbol{\varphi}_{\lambda}^{2, T}$, we infer from (4.4) that $c_{\lambda}^{T}=\sigma_{\gamma}^{2}\left(\left.\boldsymbol{u}\right|_{T}\right)$. The duality (3.14) gives then

$$
\sigma_{\gamma}^{2}\left(\mathcal{P}_{h}^{2} \boldsymbol{u}\right)=\sigma_{\gamma}^{2}\left(\sum_{\lambda \in \Lambda_{h}^{2}} \sum_{T \in \mathcal{T}_{h}(\lambda)} \frac{c_{\lambda}^{T}}{\#\left(\mathcal{T}_{h}(\lambda)\right)} \varphi_{\lambda}^{2}\right)=\frac{\sum_{T \in \mathcal{T}_{h}(\gamma)} \sigma_{\gamma}^{2}\left(\left.\boldsymbol{u}\right|_{T}\right)}{\#\left(\mathcal{T}_{h}(\gamma)\right)}, \quad \text { for } \gamma \in \Lambda_{h}^{2},
$$

hence (4.6). Now, just as the basis functions $\varphi_{\lambda}^{2, T}$ can be obtained as affine maps of reference basis functions with an $L^{2}$ normalization (3.16), it is possible to design the dual basis functions with the same property,

$$
\left\|\boldsymbol{\psi}_{\lambda}^{2, T}\right\| \sim 1 \quad \text { for } T \in \mathcal{T}_{h}, \lambda \in \Lambda^{2}(T) .
$$

In particular, $\mathcal{P}_{h}^{2}$ is locally bounded in $L^{2}$ and since it is a projection on the conforming space $V_{h}^{2}$ it satisfies an error estimate similar to (3.13).

In the analysis of the structure-preserving Conga scheme, an important tool is the adjoint operator $\left(\mathcal{P}_{h}^{2}\right)^{*}$ which is bounded on $L^{2}$ like $\mathcal{P}_{h}^{2}$, and has the form

$$
\left(\mathcal{P}_{h}^{2}\right)^{*} \boldsymbol{u}=\sum_{\lambda \in \Lambda_{h}^{2}} \sum_{T \in \mathcal{T}_{h}(\lambda)} \frac{\left\langle\boldsymbol{u}, \varphi_{\lambda}^{2}\right\rangle}{\#\left(\mathcal{T}_{h}(\lambda)\right)} \psi_{\lambda}^{2, T} .
$$

Lemma 4.1. The operator $\left(\mathcal{P}_{h}^{2}\right)^{*}$ maps on the non-conforming space $\mathcal{N}_{p-1}\left(\mathcal{T}_{h}\right)$, it is locally bounded in $L^{2}$ and it preserves the piecewise polynomials of $\mathbb{P}_{p-2}\left(\mathcal{T}_{h}\right)^{2}$. In particular, if the mesh $\mathcal{T}_{h}$ is shape regular we have

$$
\left\|\left(I-\left(\mathcal{P}_{h}^{2}\right)^{*}\right) \boldsymbol{u}\right\| \leq C h^{m}|\boldsymbol{u}|_{m}, \quad 0 \leq m \leq p-1
$$

with a constant independent of $h$.

Proof. The fact that $\left(\mathcal{P}_{h}^{2}\right)^{*}$ maps on $\mathcal{N}_{p-1}\left(\mathcal{T}_{h}\right)$ is obvious as every $\boldsymbol{\psi}_{\lambda}^{2, T}$ is in this space, and the local $L^{2}$ bound is easily derived using the localized supports of the basis functions $\varphi_{\lambda}^{2}$ and the normalization of the primal $\varphi_{\lambda}^{2, T}$ 's and the dual $\psi_{\lambda}^{2, T}$ 's, see (3.16) and (4.8). To show that $\left(\mathcal{P}_{h}^{2}\right)^{*}$ preserves the piecewise polynomials of $\mathbb{P}_{p-2}\left(\mathcal{T}_{h}\right)^{2}$ we observe that due to the form of the volume-based degrees of freedom in (3.10), the functions $\boldsymbol{\psi}_{\lambda}^{2, T}, \lambda=(T, i) \in \Lambda_{\mathrm{vol}}^{2}(T)$, coincide with the polynomials $\boldsymbol{q}_{T, i}$ in (3.14), hence they form a basis of $\mathbb{P}_{p-2}(T)^{2}$. Since the associated $\varphi_{\lambda}^{2}$ vanish outside $T$, they satisfy $\varphi_{\lambda}^{2}=\varphi_{\lambda}^{2, T}$ and it is easily seen that $\left(\mathcal{P}_{h}^{2}\right)^{*} \boldsymbol{\psi}_{\lambda}^{2, T}=\boldsymbol{\psi}_{\lambda}^{2, T}$ for all $\lambda \in \Lambda_{\text {vol }}^{2}(T)$ (note that every such $\lambda$ is in $\Lambda_{h}^{2}$ ). Estimate (4.10) is then a straightforward consequence of these properties and the Bramble-Hilbert Lemma.

\subsection{Gauss-compatibility of the non-conforming Maxwell solver}

We now establish that the above scheme can be made Gauss-compatible and give a priori error estimates leading to long-time stability. Again we denote $U_{h}=\left(c B_{h}, \boldsymbol{E}_{h}\right)^{T}$ and $F_{h}=\left(0, \varepsilon_{0}^{-1} \boldsymbol{J}_{h}\right)^{T}$. In compact form, the non-conforming Conga-Faraday scheme (4.3) reads $\partial_{t} U_{h}-\mathcal{A}_{h} U_{h}=-F_{h}$ with a 


\section{Campos Pinto \& E. Sonnendrǘker}

composite curl operator that takes a form similar to (3.17) but also involves the smoothing projection $\mathcal{P}_{h}^{2}$. Specifically, it is defined on $\mathcal{V}_{h}:=V_{h}^{1} \times \tilde{V}_{h}^{2}$ by

$$
\mathcal{A}_{h}:=c\left(\begin{array}{cc}
0 & -\operatorname{curl}_{h} \\
\operatorname{curl}_{h} & 0
\end{array}\right) \quad \text { with } \quad\left\{\begin{array}{l}
\operatorname{curl}_{h}:=\left.\operatorname{curl} \mathcal{P}_{h}^{2}\right|_{\tilde{V}_{h}^{2}}: \tilde{V}_{h}^{2} \rightarrow V_{h}^{1} \\
\operatorname{curl}_{h}:=\left(\operatorname{curl}_{h}\right)^{*}: V_{h}^{1} \rightarrow \tilde{V}_{h}^{2} .
\end{array}\right.
$$

According to Definition 2.1 and using approximation projection operators of the form (3.18), we then see that this scheme is Gauss-compatible on some product space $\hat{V}^{1} \times \hat{V}^{2}$ if for $l=1,2$ we can find approximation operators $\pi_{h}^{l}$ and $\hat{\pi}_{h}^{l}$ mapping on $V_{h}^{l}$, such that

$$
\pi_{h}^{2} \operatorname{curl} u=\left(\operatorname{curl}_{h}\right)^{*} \hat{\pi}_{h}^{1} u, \quad u \in \hat{V}^{1}
$$

and

$$
\pi_{h}^{1} \operatorname{curl} \boldsymbol{u}=\operatorname{curl} \mathcal{P}_{h}^{2} \hat{\pi}_{h}^{2} \boldsymbol{u}, \quad \boldsymbol{u} \in \hat{V}^{2} .
$$

The following compatibility result is then easy to verify.

Theorem 4.2. The Conga-Faraday scheme (4.3) associated with the corrected projection operator

$$
\pi_{h}^{2}:=\left(\mathcal{P}_{h}^{2}\right)^{*}: L^{2}(\Omega)^{2} \rightarrow \tilde{V}_{h}^{2}
$$

for the current, see (4.9), is Gauss-compatible on the product space

$$
\hat{V}^{1} \times \hat{V}^{2}:=H(\operatorname{curl} ; \Omega) \times\left(H^{1}(\Omega)^{2} \cap \boldsymbol{H}_{0}(\operatorname{curl} ; \Omega)\right) .
$$

In particular, Equation (4.12) holds with the $L^{2}$ projection $\hat{\pi}_{h}^{1}:=P_{V_{h}^{1}}$ and Equation (4.13) holds with $\pi_{h}^{1}:=P_{V_{h}^{1}}$ and $\hat{\pi}_{h}^{2}:=\pi_{h}^{\text {curl }}$ the Nédélec interpolation defined in (3.11). Moreover, these mappings satisfy

$$
\begin{array}{rlrl}
\left\|\hat{\pi}_{h}^{1} u-u\right\| & \lesssim h^{m}|u|_{m}, & & 0 \leq m \leq p \\
\left\|\hat{\pi}_{h}^{2} \boldsymbol{u}-\boldsymbol{u}\right\| \lesssim h^{m}|\boldsymbol{u}|_{m}, & & 1 \leq m \leq p, \quad \boldsymbol{u} \in \boldsymbol{H}_{0}(\operatorname{curl} ; \Omega) \\
\left\|\pi_{h}^{1} u-u\right\| & \lesssim h^{m}|u|_{m}, & & 0 \leq m \leq p \\
\left\|\pi_{h}^{2} \boldsymbol{u}-\boldsymbol{u}\right\| \lesssim h^{m}|\boldsymbol{u}|_{m}, & & 0 \leq m \leq p-1 .
\end{array}
$$

Proof. Since both sides of Equation (4.12) are in $\tilde{V}_{h}^{2}$, we can test it against an arbitrary $\boldsymbol{v} \in \tilde{V}_{h}^{2}$. We thus compute for $u \in \hat{V}^{1}=H(\operatorname{curl} ; \Omega)$

$\left\langle\pi_{h}^{2} \operatorname{curl} u, \boldsymbol{v}\right\rangle=\left\langle\left(\mathcal{P}_{h}^{2}\right)^{*} \operatorname{curl} u, \boldsymbol{v}\right\rangle=\left\langle\operatorname{curl} u, \mathcal{P}_{h}^{2} \boldsymbol{v}\right\rangle=\left\langle u, \operatorname{curl} \mathcal{P}_{h}^{2} \boldsymbol{v}\right\rangle=\left\langle P_{V_{h}^{1}} u, \operatorname{curl}_{h} \boldsymbol{v}\right\rangle=\left\langle\operatorname{curl}_{h} P_{V_{h}^{1}} u, \boldsymbol{v}\right\rangle$ where we have used the equality $\operatorname{curl}_{h}=\operatorname{curl} \mathcal{P}_{h}^{2}=\left(\operatorname{curl}_{h}\right)^{*}: \tilde{V}_{h}^{2} \rightarrow V_{h}^{1}$, and this proves (4.12) with $\hat{\pi}_{h}^{1}=P_{V_{h}^{1}}$. As for Equation (4.13), it simply follows from the commuting diagram (3.12) and the fact that we have $\operatorname{curl}_{h} \pi_{h}^{\text {curl }}=\operatorname{curl} \mathcal{P}_{h}^{2} \pi_{h}^{\text {curl }}=\operatorname{curl} \pi_{h}^{\text {curl }}$ since $\mathcal{P}_{h}^{2}$ is a projection on $V_{h}^{2}$. Estimates (4.14) and (4.16) are then standard for the $L^{2}$ projection on $V_{h}^{1}$ and (4.15), (4.17) are (3.13) and (4.10), respectively.

If one is solving the Maxwell equations with exact sources, Theorem 2.5 from [10] applies and gives the following a priori estimate.

Corollary 4.3. Let $(B, \boldsymbol{E})$ be the exact solution to the Maxwell system (2.3). The semi-discrete solution to the non-conforming Conga-Faraday scheme (4.3) coupled with the corrected projection (4.9) for the current satisfies

$$
\begin{aligned}
\left\|\left(B-B_{h}\right)(t)\right\|+\left\|\left(\boldsymbol{E}-\boldsymbol{E}_{h}\right)(t)\right\| & \lesssim\left\|B_{h}(0)-\hat{\pi}_{h}^{1} B(0)\right\|+\left\|\boldsymbol{E}_{h}(0)-\hat{\pi}_{h}^{2} \boldsymbol{E}(0)\right\| \\
& +h^{m}\left(|B(0)|_{m}+\int_{0}^{t}\left|\partial_{t} B(s)\right|_{m} \mathrm{~d} s\right)+h^{m^{\prime}}\left(|\boldsymbol{E}(0)|_{m^{\prime}}+\int_{0}^{t}\left(\left|\partial_{t} \boldsymbol{E}(s)\right|_{m^{\prime}}\right) \mathrm{d} s\right)
\end{aligned}
$$




\section{Compatible Maxwell solvers With Particles, II}

for $0 \leq m \leq p, 1 \leq m^{\prime} \leq p-1$, and with a constant independent of $h$ and $t$.

Remark 4.4. In the case of approximate sources, one must resort to the analysis developped in Section 2.4 of [10] to be able to derive long-time stability estimates. This will be done in Section 5.2 by showing that the Conga-Faraday scheme (4.3) can be equipped with a non-standard divergence that makes it structure-preserving.

\subsection{Reformulation as a standard discontinuous Galerkin scheme}

Because our smoothing projection $\mathcal{P}_{h}^{2}$ is defined as an averaged interpolation on the Nédélec elements $V_{h}^{2}$, it is possible to reformulate the Conga-Faraday scheme (4.3) as a standard DG scheme. To verify this claim we remind that a centered-flux DG approximation (see, e.g., [14]) based on the discontinuous spaces $V_{h}^{1}$ and $\tilde{V}_{h}^{2}$ defines $\left(B_{h}, \boldsymbol{E}_{h}\right) \in \mathcal{C}^{0}\left([0, T] ; V_{h}^{1} \times \tilde{V}_{h}^{2}\right)$ as the solution to

$$
\left\{\begin{array}{cl}
\left\langle\partial_{t} B_{h}, \varphi\right\rangle+\sum_{T \in \mathcal{T}_{h}}\left\langle\boldsymbol{E}_{h}, \operatorname{curl} \varphi\right\rangle_{T}-\sum_{e \in \mathcal{E}_{h} \backslash \mathcal{E}_{h}^{B}}\left\langle\left\{\left\{\boldsymbol{E}_{h}\right\}\right\}, \llbracket \varphi \rrbracket\right\rangle_{e}=0, & \varphi \in V_{h}^{1} \\
\left\langle\partial_{t} \boldsymbol{E}_{h}, \boldsymbol{\varphi}\right\rangle-\sum_{T \in \mathcal{T}_{h}} c^{2}\left\langle B_{h}, \operatorname{curl} \boldsymbol{\varphi}\right\rangle_{T}+\sum_{e \in \mathcal{E}_{h}} c^{2}\left\langle\left\{\left\{B_{h}\right\}, \llbracket \boldsymbol{\varphi} \rrbracket\right\rangle_{e}=-\frac{1}{\varepsilon_{0}}\left\langle\boldsymbol{J}_{h}, \boldsymbol{\varphi}\right\rangle,\right. & \boldsymbol{\varphi} \in \tilde{V}_{h}^{2} .
\end{array}\right.
$$

Here we have used standard notations for tangential jumps and averages (see, e.g., [6]): for interior edges (shared by two cells $T^{ \pm}=T^{ \pm}(e)$, and writing $n_{e}^{ \pm}=n_{e}^{T^{ \pm}}$for simplicity ) we denote

$$
\left.\llbracket \boldsymbol{u} \rrbracket_{e}:=\left.\left(\boldsymbol{n}_{e}^{-} \times\left.\boldsymbol{u}\right|_{T^{-}}+\boldsymbol{n}_{e}^{+} \times\left.\boldsymbol{u}\right|_{T^{+}}\right)\right|_{e} \quad \text { and } \quad\{\boldsymbol{u}\}_{e}:=\frac{1}{2}\left(\left.\boldsymbol{u}\right|_{T^{-}}+\left.\boldsymbol{u}\right|_{T^{+}}\right)\right)\left.\right|_{e} \quad \text { for } \quad e \in \mathcal{E}_{h} \backslash \mathcal{E}_{h}^{B}
$$

and for boundary edges (in the boundary of a single cell $T^{-}=T^{-}(e)$ ),

$$
\llbracket \boldsymbol{u} \rrbracket_{e}:=\left.\left(\boldsymbol{n}_{e}^{-} \times\left.\boldsymbol{u}\right|_{T^{-}}\right)\right|_{e} \quad \text { and } \quad\left\{\{\boldsymbol{u}\}_{e}:=\left.\left(\left.\boldsymbol{u}\right|_{T^{-}}\right)\right|_{e} \quad \text { for } e \in \mathcal{E}_{h}^{B} .\right.
$$

For a scalar-valued $u$ the definitions are formally the same, keeping in mind that with the $2 \mathrm{D}$ convention the product $\boldsymbol{n} \times u$ is the vector $\left(n_{y} u,-n_{x} u\right)^{T}$. To write (4.18) in an operator form we then let

$$
\operatorname{curl}_{h}^{\mathrm{DG}}: \tilde{V}_{h}^{2} \rightarrow V_{h}^{1} \quad \text { and } \quad \operatorname{curl}_{h}^{\mathrm{DG}}: V_{h}^{1} \rightarrow \tilde{V}_{h}^{2}
$$

be defined by the relations

$$
\left\{\begin{array}{l}
\left\langle\operatorname{curl}_{h}^{\mathrm{DG}} \boldsymbol{u}, v\right\rangle:=\sum_{T \in \mathcal{T}_{h}}\langle\boldsymbol{u}, \operatorname{curl} v\rangle_{T}-\sum_{e \in \mathcal{E}_{h} \backslash \mathcal{E}_{h}^{B}}\left\langle\{\boldsymbol{u} \rrbracket, \llbracket v \rrbracket\rangle_{e}\right. \\
\left\langle\operatorname{curl}_{h}^{\mathrm{DG}} v, \boldsymbol{u}\right\rangle:=\sum_{T \in \mathcal{T}_{h}}\langle v, \operatorname{curl} \boldsymbol{u}\rangle_{T}-\sum_{e \in \mathcal{E}_{h}}\left\langle\{\{v\}, \llbracket \boldsymbol{u} \rrbracket\rangle_{e}\right.
\end{array} \quad \text { for } v \in V_{h}^{1}, \boldsymbol{u} \in \tilde{V}_{h}^{2} .\right.
$$

Hence writing again $U_{h}=\left(c B_{h}, \boldsymbol{E}_{h}\right)^{T}$ and $F_{h}=\left(0, \varepsilon_{0}^{-1} \boldsymbol{J}_{h}\right)^{T}$, the DG formulation (4.18) reads

$$
\partial_{t} U_{h}-\mathcal{A}_{h} U_{h}=-F_{h} \quad \text { with } \quad \mathcal{A}_{h}:=c\left(\begin{array}{cc}
0 & -\operatorname{curl}_{h}^{\mathrm{DG}} \\
\operatorname{curl}_{h}^{\mathrm{DG}} & 0
\end{array}\right) .
$$

The following results establishes that this approximation is equivalent with the Conga method (4.3).

Theorem 4.5. The DG curl operators defined above satisfy

$$
\operatorname{curl}_{h}^{\mathrm{DG}}=\operatorname{curl} \mathcal{P}_{h}^{2} \quad \text { on } \tilde{V}_{h}^{2}, \quad \text { and } \quad \operatorname{curl}_{h}^{\mathrm{DG}}=\left(\operatorname{curl}_{h}^{\mathrm{DG}}\right)^{*} .
$$

In particular, the Conga-Faraday scheme (4.3) is equivalent with the centered-flux DG scheme (4.18). 


\section{Campos Pinto \& E. Sonnendrǘker}

Remark 4.6. This result is specific to the $2 \mathrm{D}$ setting, for several reasons. First, the different degrees involved in the edge and face degrees of freedom prevent similar computations to be carried out in 3D. In particular, it is not true that in 3D the centered DG scheme involves a strong Faraday law, as this would imply that the divergence of the magnetic field remains constant in time, a property known to be false in general [24]. Another indication is offered by considering the spectral properties of the respective methods: whereas the 3D Conga method has been proved to be spectrally correct in [11], numerical and theoretical evidences [16] show that this is not the case for the centered DG scheme.

Proof. To prove the first equality we compute for $v \in V_{h}^{1}$ and $\boldsymbol{u} \in \tilde{V}_{h}^{2}$,

$$
\begin{aligned}
\left\langle\operatorname{curl} \mathcal{P}_{h}^{2} \boldsymbol{u}, v\right\rangle & =\sum_{T \in \mathcal{T}_{h}}\left(\left\langle\mathcal{P}_{h}^{2} \boldsymbol{u}, \operatorname{curl} v\right\rangle_{T}+\left\langle\boldsymbol{n} \times \mathcal{P}_{h}^{2} \boldsymbol{u}, v\right\rangle_{\partial T}\right) \\
& =\sum_{T \in \mathcal{T}_{h}}\left(\langle\boldsymbol{u}, \operatorname{curl} v\rangle_{T}+\left\langle\boldsymbol{n} \times\{\{\boldsymbol{u}\}, v\rangle_{\partial T \backslash \partial \Omega}\right)\right. \\
& =\sum_{T \in \mathcal{T}_{h}}\langle\boldsymbol{u}, \operatorname{curl} v\rangle_{T}-\sum_{e \in \mathcal{E}_{h} \backslash \mathcal{E}_{h}^{B}}\left\langle\{\{\boldsymbol{u}\}, \llbracket v \rrbracket\rangle_{e}\right. \\
& =\left\langle\operatorname{curl}_{h}^{\mathrm{DG}} \boldsymbol{u}, v\right\rangle .
\end{aligned}
$$

Here the second equality follows from the property (4.6) of $\mathcal{P}_{h}^{2}$ and the form (3.14) of the Nédélec degrees of freedom, together with the fact that $\left.v\right|_{T} \in \mathbb{P}_{p-1}(T)$ which gives $\left.\operatorname{curl} v\right|_{T} \in \mathbb{P}_{p-2}(T)^{2}$ and $\left.v\right|_{e} \in \mathbb{P}_{p-1}(e)$ for $e \in \mathcal{E}(T)$. The desired equality $\operatorname{curl}_{h}^{\mathrm{DG}}=\operatorname{curl}_{h}^{2}$ then follows from the fact that $\operatorname{curl} \mathcal{P}_{h}^{2}$ maps on $V_{h}^{1}$. Starting next from the second line of (4.22) and integrating by parts we compute

$$
\begin{aligned}
\left\langle\operatorname{curl}_{h}^{\mathrm{DG}} v, \boldsymbol{u}\right\rangle & =\sum_{T \in \mathcal{T}_{h}}\langle v, \operatorname{curl} \boldsymbol{u}\rangle_{T}-\sum_{e \in \mathcal{E}_{h}}\left\langle\{\{v\}, \llbracket \boldsymbol{u} \rrbracket\rangle_{e}\right. \\
& =\sum_{T \in \mathcal{T}_{h}}\left(\langle\operatorname{curl} v, \boldsymbol{u}\rangle_{T}+\langle v, \boldsymbol{n} \times \boldsymbol{u}\rangle_{\partial T}\right)-\sum_{e \in \mathcal{E}_{h}}\left\langle\{\{v\}, \llbracket \boldsymbol{u} \rrbracket\rangle_{e}\right. \\
& =\sum_{T \in \mathcal{T}_{h}}\langle\boldsymbol{u}, \operatorname{curl} v\rangle_{T}+\sum_{e \in \mathcal{E}_{h} \backslash \mathcal{E}_{h}^{B}}\left(\left\langle v^{-}, \boldsymbol{n}^{-} \times \boldsymbol{u}^{-}\right\rangle_{e}+\left\langle v^{+}, \boldsymbol{n}^{+} \times \boldsymbol{u}^{+}\right\rangle_{e}-\left\langle\{\{v\}, \llbracket \boldsymbol{u} \rrbracket\rangle_{e}\right)\right. \\
& =\sum_{T \in \mathcal{T}_{h}}\langle\boldsymbol{u}, \operatorname{curl} v\rangle_{T}-\sum_{e \in \mathcal{E}_{h} \backslash \mathcal{E}_{h}^{B}}\left\langle\{\{\boldsymbol{u}\}, \llbracket v \rrbracket\rangle_{e}=\left\langle\operatorname{curl}_{h}^{\mathrm{DG}} \boldsymbol{u}, v\right\rangle\right.
\end{aligned}
$$

and the desired equality follows from the definition of the adjoint. Note that in the third equality we have used the fact that $\left\langle\{\{v\}, \llbracket \boldsymbol{u} \rrbracket\rangle_{e}=\left\langle v^{-}, \boldsymbol{n}^{-} \times \boldsymbol{u}^{-}\right\rangle_{e}\right.$ on every boundary edge $e \in \mathcal{E}_{h}^{B}$.

Remark 4.7. Discontinuous Galerkin schemes are more commonly used with standard polynomials spaces, such as

$$
\tilde{V}_{h}^{2}:=\mathbb{P}_{p-1}\left(\mathcal{T}_{h}\right)^{2}=\left\{\boldsymbol{u} \in L^{2}(\Omega):\left.\boldsymbol{u}\right|_{T} \in \mathbb{P}_{p-1}(T)^{2}, T \in \mathcal{T}_{h}\right\}
$$

To apply our analysis to that case the most natural path consists in replacing the conforming sequence (3.3) by the following one

$$
V_{h}^{3}=\mathcal{L}_{p, 0}\left(\Omega, \mathcal{T}_{h}\right) \stackrel{\left(d_{h}^{2}\right)^{*}=-\operatorname{grad}}{\longrightarrow} V_{h}^{2}=\mathbb{P}_{p-1}\left(\mathcal{T}_{h}\right)^{2} \cap \boldsymbol{H}_{0}(\operatorname{curl} ; \Omega) \stackrel{\left(d_{h}^{1}\right)^{*}=\operatorname{curl}}{\longrightarrow} V_{h}^{1}=\mathbb{P}_{p-2}\left(\mathcal{T}_{h}\right)
$$

which is also exact. Here the space $V_{h}^{2}$ corresponds to the Nédélec elements of second type. Accordingly one replaces the degrees of freedom (3.10) by

$$
\begin{cases}\mathcal{M}_{h}^{2}(T, \boldsymbol{u}):=\left\{\int_{T} \boldsymbol{u} \cdot \boldsymbol{q}: \boldsymbol{q} \in \mathbb{P}_{p-3}(T)^{2}+\left(\begin{array}{l}
x \\
y
\end{array}\right) \mathbb{P}_{p-3}(T)\right\} & \text { for every triangle } T \in \mathcal{T}_{h}, \\
\mathcal{M}_{h}^{2}(e, \boldsymbol{u}):=\left\{\int_{e}\left(\boldsymbol{n}_{e} \times \boldsymbol{u}\right) q: q \in \mathbb{P}_{p-1}(e)\right\} & \text { for every edge } e \in \mathcal{E}_{h} \backslash \mathcal{E}_{h}^{B}\end{cases}
$$




\section{Compatible Maxwell solvers With Particles, II}

see, e.g., $[22,4]$ and a new smoothing projection $\mathcal{P}_{h}^{2}$ based on these degrees of freedom can be designed following the same steps as before. One can verify that the resulting Conga scheme is equivalent with the associated DG method. Namely, Theorem 4.5 holds with $\tilde{V}_{h}^{2}=\mathbb{P}_{p-1}\left(\mathcal{T}_{h}\right)^{2}$ and $V_{h}^{1}=\mathbb{P}_{p-2}\left(\mathcal{T}_{h}\right)$. In this article we have worked with the first Nédélec space because it has better convergence properties than the second one (see e.g. [5]), but we believe that a proper theoretical and numerical study of the other option should also be performed.

\section{Application to the coupled Vlasov-Maxwell problem}

In this section we apply the new stability analysis proposed in Section 2.1 and 2.4 of [10] for approximate sources: in Section 5.1 we begin by verifying that the conforming Finite Element discretization studied in Section 3 is naturally structure-preserving in the sense of Definition 2.2, and in Section 5.2 we show that our new non-conforming Conga discretization of Section 4 is also structure-preserving, once associated with a nonstandard discrete divergence. Assuming next a discrete particle representation of the approximate current density, for each Maxwell solver we provide a charge-conserving current deposition method in the sense of Definition 2.3.

To specify the problem we consider the case where the Maxwell system is coupled with a Vlasov equation such as (1.2) involving a species of charged particles with phase space distribution function $f=f(t, \boldsymbol{x}, \boldsymbol{v})$. The charge and current densities are then given by the first moments of $f$,

$$
\rho(t, \boldsymbol{x}):=q \int f(t, \boldsymbol{x}, \boldsymbol{v}) \mathrm{d} \boldsymbol{v} \quad \text { and } \quad \boldsymbol{J}(t, \boldsymbol{x}):=q \int \boldsymbol{v} f(t, \boldsymbol{x}, \boldsymbol{v}) \mathrm{d} \boldsymbol{v} .
$$

\subsection{Structure-preserving discretization with conforming Finite Elements}

The structure-preserving properties of the conforming Maxwell scheme (3.7) essentially follow from the fact that $V_{h}^{3} \stackrel{\text { grad }}{\longrightarrow} V_{h}^{2} \stackrel{\text { curl }}{\longrightarrow} V_{h}^{1} \stackrel{f_{\Omega}}{\longrightarrow} \mathbb{R}$ is an exact sequence, as recalled in Lemma 3.2. The Poincaré estimates (2.9) are also standard to verify. The first one reads

$$
\|u\| \leq c_{P}\|\operatorname{grad} u\|, \quad u \in V_{h}^{3}
$$

and is a standard Poincaré inequality, given the homogeneous boundary condition. The second one

$$
\|\boldsymbol{u}\| \leq c_{P}\|\operatorname{curl} \boldsymbol{u}\|, \quad \boldsymbol{u} \in V_{h}^{2} \cap(\operatorname{ker} \operatorname{curl})^{\perp}
$$

can be derived, e.g. from the similar stability estimate [23, Th. 4] recalled in [10, Eq. (5.3)] for the Raviart-Thomas elements, using the standard rotation argument of Remark 3.3. Finally the third one involves the integral operator $f_{\Omega}$ and trivially holds on $V_{h}^{1} \cap\left(\operatorname{ker} f_{\Omega}\right)^{\perp} \equiv \mathbb{R}$. Hence the Lemma.

Lemma 5.1. The conforming scheme (3.7) associated with the discrete Gauss laws (2.7) defined by

$$
\left\{\begin{array}{l}
\left(\iota_{h}\right)^{*}:=f_{\Omega}: V_{h}^{1} \rightarrow \mathbb{R} \\
\operatorname{grad}_{h}:=-\left.\operatorname{grad}\right|_{V_{h}^{3}}: V_{h}^{3} \rightarrow V_{h}^{2} \\
\operatorname{div}_{h}:=-\left(\operatorname{grad}_{h}\right)^{*}: V_{h}^{2} \rightarrow V_{h}^{3}
\end{array}\right.
$$

see (3.3), is structure preserving in the sense of Definition 2.2.

Remark 5.2. With the operators (5.4), the discrete Gauss laws (2.7) read

$$
\left\{\begin{array}{l}
-\left\langle\boldsymbol{E}_{h}(t), \operatorname{grad} \phi\right\rangle=\frac{1}{\varepsilon_{0}}\left\langle\rho_{h}(t), \phi\right\rangle \quad \text { for } \phi \in V_{h}^{3} \\
f_{\Omega} B_{h}(t)=f_{\Omega} B_{h}^{0} .
\end{array}\right.
$$




\section{Campos Pinto \& E. Sonnendrǘker}

\subsection{Structure-preserving discretization with the discontinuous Conga method}

To study the structure-preserving properties of the Conga method, and identify a proper discrete divergence, we first characterize the kernel and the image of the non-conforming curl operator, following the method introduced in [7].

Lemma 5.3. The non-conforming curl operator (4.11), $\operatorname{curl}_{h}=\left.\operatorname{curl} \mathcal{P}_{h}^{2}\right|_{\tilde{V}_{h}^{2}}: \tilde{V}_{h}^{2} \rightarrow V_{h}^{1}$, satisfies

$$
\operatorname{ker}\left(\operatorname{curl}_{h}\right)=\operatorname{grad} V_{h}^{3} \oplus\left(I-\mathcal{P}_{h}^{2}\right) \tilde{V}_{h}^{2} \quad \text { and } \quad \operatorname{Im}\left(\operatorname{curl}_{h}\right)=V_{h}^{1} \cap \mathbb{R}^{\perp} .
$$

Proof. Starting with the first identity, the inclusion $\supset$ is verified by applying $\operatorname{curl} \mathcal{P}_{h}^{2}$ and the fact that $\operatorname{grad} V_{h}^{3}$ is a subset of $V_{h}^{2}$ where $\mathcal{P}_{h}^{2}=I$. To verify the inclusion $\subset$ we take $\boldsymbol{u} \in \tilde{V}_{h}^{2} \cap \operatorname{ker}\left(\operatorname{curl} \mathcal{P}_{h}^{2}\right)$. Then $\mathcal{P}_{h}^{2} u$ is in $V_{h}^{2} \cap$ ker curl which coincides with grad $V_{h}^{3}$ thanks to Lemma 3.2. Hence we have

$$
\boldsymbol{u}=\mathcal{P}_{h}^{2} \boldsymbol{u}+\left(I-\mathcal{P}_{h}^{2}\right) \boldsymbol{u} \in \operatorname{grad} V_{h}^{3} \oplus\left(I-\mathcal{P}_{h}^{2}\right) \tilde{V}_{h}^{2},
$$

and we easily verify that this is an orthogonal sum. The second identity follows from Lemma 3.2 and the fact that $\tilde{V}_{h}^{2}$ contains $V_{h}^{2}$, hence $\mathcal{P}_{h}^{2} \tilde{V}_{h}^{2}=V_{h}^{2}$.

We are then in position to establish that the Conga-Faraday scheme is structure preserving when associated with the proper discrete operators for the Gauss laws.

Lemma 5.4. The non-conforming Conga scheme (4.3) associated with the discrete Gauss laws (2.7) defined by

$$
\left\{\begin{array}{l}
\left(\iota_{h}\right)^{*}:=f_{\Omega}: V_{h}^{1} \rightarrow \mathbb{R} \\
\operatorname{grad}_{h}:\left(V_{h}^{3} \times \tilde{V}_{h}^{2}\right) \ni(\phi, \tilde{\boldsymbol{u}}) \mapsto \operatorname{grad} \phi+\left(I-\mathcal{P}_{h}^{2}\right) \tilde{\boldsymbol{u}} \in \tilde{V}_{h}^{2} \\
\operatorname{div}_{h}:=-\left(\operatorname{grad}_{h}\right)^{*}: \tilde{V}_{h}^{2} \rightarrow\left(V_{h}^{3} \times \tilde{V}_{h}^{2}\right)
\end{array}\right.
$$

see (3.3), is structure preserving in the sense of Definition 2.2.

Remark 5.5. With the proposed operators (5.6), the discrete Gauss laws (2.7) read

$$
\left\{\begin{array}{l}
-\left\langle\boldsymbol{E}_{h}(t),\left(\operatorname{grad} \phi+\left(I-\mathcal{P}_{h}^{2}\right) \tilde{\boldsymbol{u}}\right)\right\rangle=\frac{1}{\varepsilon_{0}}\left\langle\rho_{h}(t), \phi\right\rangle \quad \text { for }(\phi, \tilde{\boldsymbol{u}}) \in V_{h}^{3} \times \tilde{V}_{h}^{2} \\
f_{\Omega} B_{h}(t)=f_{\Omega} B_{h}^{0} .
\end{array}\right.
$$

Proof. Here the exact sequence property $(2.8)$ reads

$$
V_{h}^{3} \times \tilde{V}_{h}^{2} \stackrel{\operatorname{grad}_{h}}{\longrightarrow} \tilde{V}_{h}^{2} \stackrel{\operatorname{curl}_{h}=\operatorname{curl}_{h}^{2}}{\longrightarrow} V_{h}^{1} \stackrel{f_{\Omega}}{\longrightarrow} \mathbb{R}
$$

and it follows from Lemma 5.3. To prove the stability estimates in (2.9) we follow the proof of Theorem 4.1 from [8]. We begin by observing that since $\operatorname{grad} V_{h}^{3} \oplus\left(I-\mathcal{P}_{h}^{2}\right) \tilde{V}_{h}^{2}$ is a direct sum, one has

$$
\text { ker } \operatorname{grad}_{h}=\left(V_{h}^{3} \cap \operatorname{ker} \operatorname{grad}\right) \times\left(\tilde{V}_{h}^{2} \cap \operatorname{ker}\left(I-\mathcal{P}_{h}^{2}\right)\right)=\{0\} \times V_{h}^{2} .
$$

Considering then $\phi \in V_{h}^{3}, \tilde{\boldsymbol{u}} \in \tilde{V}_{h}^{2} \cap\left(V_{h}^{2}\right)^{\perp}$ and using (5.2) we compute

$$
\begin{aligned}
\|(\phi, \tilde{\boldsymbol{u}})\|^{2} \leq c_{P}\|\operatorname{grad} \phi\|^{2}+\|\tilde{\boldsymbol{u}}\|^{2} & \lesssim\left\|\operatorname{grad} \phi-\mathcal{P}_{h}^{2} \tilde{\boldsymbol{u}}\right\|^{2}+\left\|\mathcal{P}_{h}^{2} \tilde{\boldsymbol{u}}\right\|^{2}+\|\tilde{\boldsymbol{u}}\|^{2} \\
& \lesssim\left\|\operatorname{grad} \phi-\mathcal{P}_{h}^{2} \tilde{\boldsymbol{u}}\right\|^{2}+\|\tilde{\boldsymbol{u}}\|^{2}=\left\|\operatorname{grad} \phi+\left(I-\mathcal{P}_{h}^{2}\right) \tilde{\boldsymbol{u}}\right\|^{2}
\end{aligned}
$$

where the last equality uses that $\operatorname{grad} \phi-\mathcal{P}_{h}^{2} \tilde{\boldsymbol{u}}$ is in $V_{h}^{2}$ and hence is orthogonal to $\tilde{\boldsymbol{u}}$. This is the first estimate in (2.9). For the second estimate, we use again the identity $\operatorname{ker}^{\operatorname{curl}_{h}}=\operatorname{grad} V_{h}^{3} \oplus\left(I-\mathcal{P}_{h}^{2}\right) \tilde{V}_{h}^{2}$ and consider now

$$
\tilde{\boldsymbol{u}} \in \tilde{V}_{h}^{2} \cap\left(\operatorname{ker}_{\operatorname{curl}}\right)^{\perp}=\tilde{V}_{h}^{2} \cap\left(\operatorname{grad} V_{h}^{3}\right)^{\perp} \cap\left(\left(I-\mathcal{P}_{h}^{2}\right) \tilde{V}_{h}^{2}\right)^{\perp}
$$




\section{Compatible Maxwell solvers With Particles, II}

and let $\boldsymbol{u} \in V_{h}^{2} \cap(\operatorname{ker} \operatorname{curl})^{\perp}$ be defined by $\operatorname{curl} \boldsymbol{u}=\operatorname{curl} \mathcal{P}_{h}^{2} \tilde{\boldsymbol{u}}$. This implies that the difference $\boldsymbol{u}-\mathcal{P}_{h}^{2} \tilde{\boldsymbol{u}}$ is in $V_{h}^{2} \cap \operatorname{ker} \operatorname{curl}=\operatorname{grad} V_{h}^{3}$, hence it is orthogonal to $\tilde{\boldsymbol{u}}$. Because the latter is also orthogonal to $\left(I-\mathcal{P}_{h}^{2}\right) \tilde{\boldsymbol{u}}$, we find that it is orthogonal to $\tilde{\boldsymbol{u}}-\boldsymbol{u}$. Using this and the conforming Poincaré estimate (5.3) for $\boldsymbol{u}$ we compute

$$
\|\tilde{\boldsymbol{u}}\| \leq\left(\|\tilde{\boldsymbol{u}}\|^{2}+\|\boldsymbol{u}-\tilde{\boldsymbol{u}}\|^{2}\right)^{\frac{1}{2}}=\|\boldsymbol{u}\| \leq c_{P}\|\operatorname{curl} \boldsymbol{u}\|=c_{P}\left\|\operatorname{curl} \mathcal{P}_{h}^{2} \tilde{\boldsymbol{u}}\right\|
$$

which proves the non-conforming Poincaré estimate. Finally the third estimate is the same as in the conforming case, and the proof is complete.

\subsection{Charge-conserving coupling with smooth particles}

In the particle method the phase-space distribution function $f$ solution to (1.2) is approached by a sum of $N$ (macro) particles with positions $\boldsymbol{x}_{\kappa}(t)$ and velocities $\boldsymbol{v}_{\kappa}(t)=\boldsymbol{x}_{\kappa}^{\prime}(t), \kappa=1, \ldots N$, that are pushed forward along the integral curves of the semi-discrete force field computed by the Maxwell scheme, using some given ODE solver. The approximated density is then

$$
f_{N}(t, \boldsymbol{x}, \boldsymbol{v})=\sum_{\kappa=1}^{N} q_{\kappa} \zeta_{\varepsilon}\left(\boldsymbol{x}-\boldsymbol{x}_{\kappa}(t)\right) \zeta_{\varepsilon}\left(\boldsymbol{v}-\boldsymbol{v}_{\kappa}(t)\right)
$$

where $q_{\kappa}$ is the numerical charge associated with the $\kappa$-th (macro) particle and $\zeta_{\varepsilon}$ is a shape function supported in the Ball $B(0, \varepsilon)$ of center 0 and radius $\varepsilon \geq 0$, which can either be a smooth approximation of the Dirac measure if $\varepsilon>0$ (typically a spline with unit mass, see e.g. [18]) or the Dirac measure itself if $\varepsilon=0$. The corresponding approximations for the charge and current densities read then

$$
\rho_{N}(t, \boldsymbol{x}):=\sum_{\kappa=1}^{N} q_{\kappa} \zeta_{\varepsilon}\left(\boldsymbol{x}-\boldsymbol{x}_{\kappa}(t)\right) \quad \text { and } \quad \boldsymbol{J}_{N}(t, \boldsymbol{x}):=\sum_{\kappa=1}^{N} q_{\kappa} \boldsymbol{v}_{\kappa}(t) \zeta_{\varepsilon}\left(\boldsymbol{x}-\boldsymbol{x}_{\kappa}(t)\right) .
$$

We observe that since $\boldsymbol{v}_{\kappa}(t)=\boldsymbol{x}_{\kappa}^{\prime}(t)$, these particle densities satisfy an exact continuity equation,

$$
\operatorname{div} \boldsymbol{J}_{N}=\sum_{\kappa=1}^{N} q_{\kappa} \operatorname{div}\left(\boldsymbol{v}_{\kappa} \zeta_{\varepsilon}\left(\cdot-\boldsymbol{x}_{\kappa}\right)\right)=\sum_{\kappa=1}^{N} q_{\kappa} \boldsymbol{v}_{\kappa} \cdot \operatorname{grad} \zeta_{\varepsilon}\left(\cdot-\boldsymbol{x}_{\kappa}\right)=-\sum_{\kappa=1}^{N} q_{\kappa} \partial_{t} \zeta_{\varepsilon}\left(\cdot-\boldsymbol{x}_{\kappa}\right)=-\partial_{t} \rho_{N} .
$$

Note that in the case where $\varepsilon=0$ these equalities hold in a weak sense, as we have

$$
\left\langle\boldsymbol{J}_{N}, \operatorname{grad} \phi\right\rangle=\sum_{\kappa=1}^{N} q_{\kappa} \boldsymbol{v}_{\kappa}(t) \cdot \operatorname{grad} \phi\left(\boldsymbol{x}_{\kappa}(t)\right)=\sum_{\kappa=1}^{N} q_{\kappa} \partial_{t} \phi\left(\boldsymbol{x}_{\kappa}(t)\right)=\sum_{\kappa=1}^{N} q_{\kappa}\left\langle\partial_{t} \delta_{\boldsymbol{x}_{\kappa}(t)}, \phi\right\rangle=\left\langle\partial_{t} \rho_{N}, \phi\right\rangle
$$

for $\phi \in \mathcal{C}^{2}(\Omega)$

In order to make both the conforming and the non-conforming schemes charge conserving in the sense of Definition 2.3 we must then find proper approximations $\boldsymbol{J}_{h}$ for the particle current $\boldsymbol{J}_{N}$. The following result shows that for this task we can use the orthogonal projection in the conforming case and the corrected projection in the non-conforming case, just as for the compatibility results stated in Sections 3.4 and 4.2 .

Theorem 5.6. Let $\varepsilon>0$. The respective conforming (FEM) and non-conforming (Conga) schemes (3.7) and (4.3), associated with the discrete Gauss laws (2.7) defined by the discrete divergence operators (5.4) and (5.6) respectively, are charge conserving in the sense of Definition 2.3 when the discrete sources are defined from the particle charge and current densities (5.10) by

$$
\rho_{h}(t):=P_{V_{h}^{3}} \rho_{N}(t) \in V_{h}^{3} \quad \text { and } \quad \boldsymbol{J}_{h}(t):=P_{V_{h}^{2}} \boldsymbol{J}_{N}(t) \in V_{h}^{2}
$$

in the conforming case and

$$
\rho_{h}(t):=P_{V_{h}^{3}} \rho_{N}(t) \in V_{h}^{3} \quad \text { and } \quad \boldsymbol{J}_{h}(t):=\left(\mathcal{P}_{h}^{2}\right)^{*} \boldsymbol{J}_{N}(t) \in \tilde{V}_{h}^{2}
$$




\section{Campos Pinto \& E. Sonnendrǘker}

in the non-conforming case. Here $P_{V_{h}^{3}}$ and $P_{V_{h}^{2}}$ are the $L^{2}$ (orthogonal) projections on the continuous and Nédélec spaces respectively, and $\left(\mathcal{P}_{h}^{2}\right)^{*}$ is the discrete adjoint of the smoothing projector, see (4.9).

Remark 5.7. As will be explained in Section 5.5, the compatible current deposition proposed here for the Conga method only involves local computations. This is a significant difference with the conforming case where the $L^{2}$ projection requires to invert a mass matrix in the curl-conforming space $V_{h}^{2}$, which is a global operation.

Proof. Since we already know that (3.7) and (4.3) are structure preserving when associated with the respective operators (5.4) and (5.6), it suffices to verify that the resulting discrete continuity equation (2.15) indeed holds in both cases. In the conforming case where $\operatorname{div}_{h}$ is defined by its adjoint $\left(\operatorname{div}_{h}\right)^{*}=\operatorname{grad}: V_{h}^{3} \rightarrow V_{h}^{2}$ the discrete continuity equation reads

$$
\left\langle\boldsymbol{J}_{h}, \operatorname{grad} \phi\right\rangle=\left\langle\partial_{t} \rho_{h}, \phi\right\rangle \quad \text { for } \phi \in V_{h}^{3}
$$

and is easily verified for $\varepsilon>0$ by computing as in (5.12) with $\phi \in V_{h}^{3}$, since then both $\boldsymbol{J}_{N}(t)$ and $\rho_{N}(t)$ are in $L^{2}(\Omega)$. In the non-conforming case the discrete operator $\operatorname{div}_{h}$ is defined by its adjoint $\left(\operatorname{div}_{h}\right)^{*}=\operatorname{grad}_{h}: V_{h}^{3} \times \tilde{V}_{h}^{2} \rightarrow \tilde{V}_{h}^{2}$ as in (5.6), and the discrete continuity equation reads

$$
\left\langle\boldsymbol{J}_{h}, \operatorname{grad}_{h}(\phi, \tilde{\boldsymbol{u}})\right\rangle=\left\langle\boldsymbol{J}_{h}, \operatorname{grad} \phi+\left(I-\mathcal{P}_{h}^{2}\right) \tilde{\boldsymbol{u}}\right\rangle=\left\langle\partial_{t} \rho_{h}, \phi\right\rangle \quad \text { for }(\phi, \tilde{\boldsymbol{u}}) \in V_{h}^{3} \times \tilde{V}_{h}^{2} .
$$

In particular, plugging $\boldsymbol{J}_{h}=\left(\mathcal{P}_{h}^{2}\right)^{*} \boldsymbol{J}_{N}$ in the above formula and using the fact that grad $V_{h}^{3} \subset V_{h}^{2}$ yields $\left\langle\boldsymbol{J}_{h}, \operatorname{grad}_{h}(\phi, \tilde{\boldsymbol{u}})\right\rangle=\left\langle\boldsymbol{J}_{N}, \mathcal{P}_{h}^{2} \operatorname{grad} \phi\right\rangle=\left\langle\boldsymbol{J}_{N}, \operatorname{grad} \phi\right\rangle$, so that the desired equality follows as in the conforming case.

\subsection{Charge-conserving coupling with point particles}

To extend our results to the case of point particles $(\varepsilon=0)$ it is convenient to consider a fully discrete version of the proposed Maxwell solvers. For simplicity we assume an explicit leap-frog time scheme. For the conforming (FEM) method (3.7) the approximate fields $\left(B_{h}^{n+1 / 2}, \boldsymbol{E}_{h}^{n}\right) \in V_{h}^{1} \times V_{h}^{2}$ are then given by

$$
\left\{\begin{array}{cc}
B_{h}^{n+\frac{1}{2}}-B_{h}^{n-\frac{1}{2}}+\Delta t \operatorname{curl} \boldsymbol{E}_{h}^{n}=0 & \left(\text { in } V_{h}^{1}\right) \\
\left\langle\boldsymbol{E}_{h}^{n+1}-\boldsymbol{E}_{h}^{n}, \boldsymbol{\varphi}\right\rangle-c^{2} \Delta t\left\langle B_{h}^{n+\frac{1}{2}}, \operatorname{curl} \boldsymbol{\varphi}\right\rangle=-\frac{\Delta t}{\varepsilon_{0}}\left\langle\boldsymbol{J}_{h}^{n+\frac{1}{2}}, \boldsymbol{\varphi}\right\rangle & \boldsymbol{\varphi} \in V_{h}^{2}
\end{array}\right.
$$

and for the non-conforming (Conga) method (4.3) the discrete fields $\left(B_{h}^{n+1 / 2}, \boldsymbol{E}_{h}^{n}\right) \in V_{h}^{1} \times \tilde{V}_{h}^{2}$ are updated with

$$
\left\{\begin{array}{cc}
B_{h}^{n+\frac{1}{2}}-B_{h}^{n-\frac{1}{2}}+\Delta t \operatorname{curl} \mathcal{P}_{h}^{2} \boldsymbol{E}_{h}^{n}=0 & \text { (in } \left.V_{h}^{1}\right) \\
\left\langle\boldsymbol{E}_{h}^{n+1}-\boldsymbol{E}_{h}^{n}, \tilde{\boldsymbol{\varphi}}\right\rangle-c^{2} \Delta t\left\langle B_{h}^{n+\frac{1}{2}}, \operatorname{curl} \mathcal{P}_{h}^{2} \tilde{\boldsymbol{\varphi}}\right\rangle=-\frac{\Delta t}{\varepsilon_{0}}\left\langle\boldsymbol{J}_{h}^{n+\frac{1}{2}}, \tilde{\boldsymbol{\varphi}}\right\rangle & \tilde{\boldsymbol{\varphi}} \in \tilde{V}_{h}^{2} .
\end{array}\right.
$$

In both cases we thus need to define $\boldsymbol{J}_{h}^{n+1 / 2}$ from the current density $\boldsymbol{J}_{N}$ carried by the moving particles. Following our stability analysis we would like that the resulting solutions satisfy the proper discrete Gauss laws which involve the structure-preserving divergence operators identified in this work, namely (5.5) and (5.7) respectively. In [9] this construction was described for the conforming FEM method, using a time averaging and an extension of the $L^{2}$ projection (5.13) for current densities carried by Dirac particles. Specifically, it was shown that the quantities

$$
\left\langle\boldsymbol{J}_{h}^{n+\frac{1}{2}}, \boldsymbol{\varphi}\right\rangle=\left\langle\int_{t^{n}}^{t^{n+1}} \boldsymbol{J}_{N}(\tau) \frac{\mathrm{d} \tau}{\Delta t}, \boldsymbol{\varphi}\right\rangle=\sum_{\kappa=1}^{N} q_{\kappa} \int_{t^{n}}^{t^{n+1}} \boldsymbol{v}_{\kappa}(\tau) \cdot \boldsymbol{\varphi}\left(\boldsymbol{x}_{\kappa}(\tau)\right) \frac{\mathrm{d} \tau}{\Delta t}, \quad \boldsymbol{\varphi} \in V_{h}^{2},
$$




\section{Compatible Maxwell solvers With Particles, II}

are well defined for point particles, and allow to define a current $\boldsymbol{J}_{h}^{n+1 / 2} \in V_{h}^{2}$ which satisfies a time-discrete version of the proper continuity equation (5.15) in the conforming case, i.e.,

$$
\left\langle\boldsymbol{J}_{h}^{n+\frac{1}{2}}, \operatorname{grad} \phi\right\rangle=\frac{1}{\varepsilon_{0}}\left\langle\frac{1}{\Delta t}\left(\rho_{h}^{n+1}-\rho_{h}^{n}\right), \phi\right\rangle \quad \phi \in V_{h}^{3} .
$$

Here $\rho_{h}^{n} \in V_{h}^{3}$ is defined by the relations

$$
\left\langle\rho_{h}^{n}, \phi\right\rangle=\left\langle\rho_{N}\left(t^{n}\right), \phi\right\rangle=\sum_{\kappa=1}^{N} q_{\kappa} \phi\left(\boldsymbol{x}_{\kappa}\left(t^{n}\right)\right), \quad \phi \in V_{h}^{3} .
$$

Essentially, the reason why (5.19) makes sense (and is stable with respect to the particle trajectories) is that the test functions $\varphi$ in the curl-conforming space $V_{h}^{2}$ have their tangential components that are continuous across interelement edges. In particular, we observe that in the last integral the discontinuities of $\boldsymbol{\varphi}$ may only pose a problem when the particle trajectory $\boldsymbol{x}_{\kappa}$ runs along an edge during some non-zero time interval. But in this case the particle velocity is tangent to the edge and the function $\boldsymbol{v}_{\kappa}(\tau) \cdot \boldsymbol{\varphi}\left(\boldsymbol{x}_{\kappa}(\tau)\right)$ is well defined, i.e., stable with respect to the trajectory.

For the same reason, it is possible to extend the corrected projection $\left(\mathcal{P}_{h}^{2}\right)^{*}$ in $(5.14)$ when the current is carried by point particles. Specifically, for the fully discrete Conga scheme (5.18) we define a charge-conserving current $\boldsymbol{J}_{h}^{n+1 / 2} \in \tilde{V}_{h}^{2}$ by the relations

$$
\left\langle\boldsymbol{J}_{h}^{n+\frac{1}{2}}, \tilde{\boldsymbol{\varphi}}\right\rangle=\left\langle\int_{t^{n}}^{t^{n+1}} \boldsymbol{J}_{N}(\tau) \frac{\mathrm{d} \tau}{\Delta t}, \mathcal{P}_{h}^{2} \tilde{\boldsymbol{\varphi}}\right\rangle=\sum_{\kappa=1}^{N} q_{\kappa} \int_{t^{n}}^{t^{n+1}} \boldsymbol{v}_{\kappa}(\tau) \cdot\left(\mathcal{P}_{h}^{2} \tilde{\boldsymbol{\varphi}}\right)\left(\boldsymbol{x}_{\kappa}(\tau)\right) \frac{\mathrm{d} \tau}{\Delta t}, \quad \tilde{\boldsymbol{\varphi}} \in \tilde{V}_{h}^{2}
$$

since then $\mathcal{P}_{h}^{2} \tilde{\boldsymbol{\varphi}}$ is curl-conforming although $\tilde{\boldsymbol{\varphi}}$ was fully discontinuous. Notice that an orthogonal projection on the fully discontinuous space $\tilde{V}_{h}^{2}$ would involve products of the form $\int \boldsymbol{v}_{\kappa}(\tau) \cdot \tilde{\boldsymbol{\varphi}}\left(\boldsymbol{x}_{\kappa}(\tau)\right) \mathrm{d} \tau$ which are not well-defined for particles running along the edges of the mesh. Arguing next as in the proof of Theorem 5.6, one easily verifies that the resulting sources satisfy indeed the proper continuity equation (5.16), namely

$$
\left\langle\boldsymbol{J}_{h}^{n+\frac{1}{2}}, \operatorname{grad} \phi+\left(I-\mathcal{P}_{h}^{2}\right) \tilde{\boldsymbol{u}}\right\rangle=\frac{1}{\varepsilon_{0}}\left\langle\frac{1}{\Delta t}\left(\rho_{h}^{n+1}-\rho_{h}^{n}\right), \phi\right\rangle \quad(\phi, \tilde{\boldsymbol{u}}) \in V_{h}^{3} \times \tilde{V}_{h}^{2}
$$

with $\rho_{h}^{n} \in V_{h}^{3}$ defined again by (5.21).

Remark 5.8. When the particle trajectories are piecewise polynomials as is usually the case, it is possible to compute exactly the time integrals in (5.19) and (5.22) using Gauss quadratures that involve a few points within each cell travelled by the particles. We refer to [9] for the detailed algorithms.

\subsection{Compatible current deposition seen as a correction method}

Before turning to the numerical experiments, let us make two simple but important observations. First, given a cell-wise basis for $\tilde{V}_{h}^{2}$ that we may denote as $\varphi_{T, \lambda}$ with $T \in \mathcal{T}_{h}$ and $\lambda \in \Lambda^{2}(T)$, we find that the coefficients $J_{T, \lambda}$ of the compatible current $\boldsymbol{J}_{h}:=\left(\mathcal{P}_{h}^{2}\right)^{*} \boldsymbol{J}_{N}$ are determined by the relations

$$
\sum_{\lambda \in \Lambda^{2}(T)} J_{T, \lambda}\left\langle\boldsymbol{\varphi}_{T, \lambda}, \boldsymbol{\varphi}_{T, \gamma}\right\rangle=\left\langle\boldsymbol{J}_{h}, \boldsymbol{\varphi}_{T, \gamma}\right\rangle=\left\langle\boldsymbol{J}_{N}, \mathcal{P}_{h}^{2} \boldsymbol{\varphi}_{T, \gamma}\right\rangle \quad \text { for } T \in \mathcal{T}_{h}, \gamma \in \Lambda^{2}(T) .
$$

Thus, the proposed deposition method involves (i) computing the products of the smooth particle current against the averaged basis functions, and (ii) inverting the local mass matrices associated with the discontinuous basis, namely

$$
\mathbf{M}_{T}=\left(\left\langle\boldsymbol{\varphi}_{T, \lambda}, \boldsymbol{\varphi}_{T, \gamma}\right\rangle\right)_{\lambda, \gamma \in \Lambda^{2}(T)}
$$




\section{Campos Pinto \& E. Sonnendrǘker}

In particular we see that these two steps can be performed locally, unlike in the conforming case (5.13) where the inversion of a mass matrix of $V_{h}^{2}$ is always a global operation over the mesh.

Second, we observe that step (i) above is easily obtained from the standard DG current coefficients when they are available in an existing code. Here we refer to the current defined by a standard $L^{2}$ projection on the fully discontinuous space, namely

$$
\boldsymbol{J}_{h}^{\mathrm{nc}}:=P_{\tilde{V}_{h}^{2}} \boldsymbol{J}_{N}
$$

where the exponent "nc" stands for "non-compatible" as this projection does not satisfy the discrete continuity equation that we have identified in the non-conforming case. The coefficients $J_{T, \lambda}^{\text {nc }}$ of the latter in the cell-wise basis are then determined by the relations

$$
\sum_{\lambda \in \Lambda^{2}(T)} J_{T, \lambda}^{\mathrm{nc}}\left\langle\boldsymbol{\varphi}_{T, \lambda}, \boldsymbol{\varphi}_{T, \gamma}\right\rangle=\left\langle\boldsymbol{J}_{h}^{\mathrm{nc}}, \boldsymbol{\varphi}_{T, \gamma}\right\rangle=\left\langle\boldsymbol{J}_{N}, \boldsymbol{\varphi}_{T, \gamma}\right\rangle .
$$

Now, since the averaging projection $\mathcal{P}_{h}^{2}$ maps on $V_{h}^{2}$ which is a subspace of $\tilde{V}_{h}^{2}$ by construction, it can be represented by a matrix $\mathbf{P}$ satisfying

$$
\mathcal{P}_{h}^{2} \boldsymbol{\varphi}_{T, \lambda}=\sum_{T^{\prime} \in \mathcal{T}_{h}, \gamma \in \Lambda^{2}\left(T^{\prime}\right)} \mathbf{P}_{(T, \lambda),\left(T^{\prime}, \gamma\right)} \boldsymbol{\varphi}_{T^{\prime}, \gamma}
$$

so that the compatible moments $m_{T, \lambda}\left(\boldsymbol{J}_{N}\right):=\left\langle\boldsymbol{J}_{N}, \mathcal{P}_{h}^{2} \boldsymbol{\varphi}_{T, \gamma}\right\rangle$ are easily derived from their standard (non-compatible) counterparts $m_{T, \lambda}^{\text {nc }}\left(\boldsymbol{J}_{N}\right):=\left\langle\boldsymbol{J}_{N}, \boldsymbol{\varphi}_{T, \gamma}\right\rangle$ as

$$
m_{T, \lambda}\left(\boldsymbol{J}_{N}\right)=\sum_{T^{\prime}, \gamma} \mathbf{P}_{(T, \lambda),\left(T^{\prime}, \gamma\right)} m_{T^{\prime}, \gamma}^{\mathrm{nc}}\left(\boldsymbol{J}_{N}\right)
$$

If the standard moments $m^{\text {nc }}$ of the particle current are available, the above formula suffices to perform step (i) and deposit the current in a compatible way. We may also verify that the coefficients of the compatible current can be expressed as a local correction of the non-compatible ones, without referring to the moments themselves: using global notations for arrays and matrices defined over the whole mesh, Equation (5.25) reads $\mathbf{M} J^{\mathrm{nc}}=m^{\mathrm{nc}}\left(\boldsymbol{J}_{N}\right)$, whereas (5.24) and (5.26) give $\mathbf{M} J=m\left(\boldsymbol{J}_{N}\right)=\mathbf{P} m^{\mathrm{nc}}\left(\boldsymbol{J}_{N}\right)$. Therefore we have

$$
J=\mathbf{M}^{-1} \mathbf{P} \mathbf{M} J^{\mathrm{nc}} .
$$

Here the matrix $\mathbf{M}$ has a block diagonal structure corresponding to the mesh cells, whereas $\mathbf{P}$ has entries corresponding to couples of adjacent cells, in addition to those on the diagonal. In particular its application is indeed a local operation, with coefficients easily given by (4.5): using local bases for the broken Nédélec spaces that derive from the curl-conforming ones as described in Section 3.3, the non-zero entries of $\mathbf{P}$ are simply 1 for the volume-based degrees of freedom and $\frac{1}{2}$ for the adjacent edge-based degrees of freedom.

\section{Numerical results}

In this section we illustrate the proposed FEM and Conga methods on the two test cases already used in our companion article [10]. For the time discretization we use the explicit leap-frog scheme described in Section 5.4.

Remark 6.1. When studying the Conga method we have observed that the smoothed field $\mathcal{P}_{h}^{2} \boldsymbol{E}_{h}^{n}$ was more accurate than the discontinuous field $\boldsymbol{E}_{h}^{n}$ itself: for the studied cases, it had smaller errors and higher convergence rates. Therefore we have decided to use the smoothing projection $\mathcal{P}_{h}^{2}$ as a systematic post-processing filter. Since this is a local operation on the discrete fields, its effect on the overall computational time is not significant. 


\section{Compatible Maxwell solvers With Particles, II}

\subsection{A Pure Maxwell problem: the 2D Issautier test case}

To assess the basic convergence and stability properties of the proposed schemes we use the analytical current source proposed in $[17,12]$ to study the charge conservation properties of a penalized finite volume scheme. The problem is posed in a metallic cavity $\Omega=[0,1]^{2}$ with articifial permittivity $\varepsilon_{0}$ and light speed $c$ equal to one, and the current density is given as

$$
\boldsymbol{J}(t, x, y)=(\cos (t)-1)\left(\begin{array}{l}
\pi \cos (\pi x)+\pi^{2} x \sin (\pi y) \\
\pi \cos (\pi y)+\pi^{2} y \sin (\pi x)
\end{array}\right)-\cos (t)\left(\begin{array}{l}
x \sin (\pi y) \\
y \sin (\pi x)
\end{array}\right) .
$$

We consider initial fields $\boldsymbol{E}^{0}=0$ and $B^{0}=0$, so that the exact solution is

$$
\left\{\begin{array}{l}
\boldsymbol{E}(t, x, y)=\sin (t)\left(\begin{array}{l}
x \sin (\pi y) \\
y \sin (\pi x)
\end{array}\right) \\
B(t, x, y)=(\cos (t)-1)(\pi y \cos (\pi x)-\pi x \cos (\pi y)) .
\end{array}\right.
$$

We note that the associated charge density reads then $\rho(t, x, y)=\sin (t)(\sin (\pi x)+\sin (\pi y))$.

In Figure 6.1 we first assess the convergence properties of the two proposed methods by plotting the relative $L^{2}$ errors $e_{h}:=\max \left(\left\|\boldsymbol{E}-\boldsymbol{E}_{h}\right\| /\|\boldsymbol{E}\|,\left\|B-B_{h}\right\| /\|B\|\right)$ at time $t=0.2 \pi$. In the left plot we show the results obtained with the conforming FEM (3.7) using different degrees and in the right plot we show the errors corresponding to the non-conforming Conga method (4.3). The convergence rates of both the FEM and Conga solutions is in agreement with Corollary 3.5 and 4.3. More precisely, we observe that the Conga solutions of degree $p+1$ (smoothed as described in Remark 6.1) converge with a similar rate than the conforming solutions of degree $p$, close to $h^{p}$. We also note that the former has higher accuracy.

Time wise, we have observed that with our straightforward implementation the Conga simulations were more efficient than the FEM ones when the meshes became finer, which is not surprising since the former is purely local and does not require any global matrix inversion. Specifically, our simulations have shown that for $p>1$, the computational time of the FEM method with degree $p$ becomes higher than that of the Conga method with degree $p+1$, as soon as the mesh has more than about 6000 triangles (which corresponds to $h \leq 0.06$ for the meshes used here).

In order to assess the long-time properties of the Conga scheme (4.3) associated with a corrected projection for the current density,

$$
\boldsymbol{J}_{h}=\left(\mathcal{P}_{h}^{2}\right)^{*} \boldsymbol{J} \quad \in \tilde{V}_{h}^{2}
$$

as supported by our analysis, we have plotted in the left panel of Figure 6.2 the $L^{2}$ norm of an electric field computed with that method. On the right panel we have shown the norm of the electric field obtained with the same scheme (4.3) but with a discrete current density computed by an orthogonal projection on the broken Nédélec space $\tilde{V}_{h}^{2}$, namely

$$
\boldsymbol{J}_{h}=P_{\tilde{V}_{h}^{2}} \boldsymbol{J} \quad \in \tilde{V}_{h}^{2}
$$

In both cases the broken Nédélec space (4.1) is defined with $p=2$, using a mesh with about 250 triangles $(h \approx 0.3)$.

In the latter case a rapid (linear in time) deterioration of the solution is visible, but with the Gausscompatible scheme the solution is stable, as predicted by Corollaries 2.6 and 2.7 in [10], applied to the constant and time-harmonic parts of the Issautier field (6.2). Note that here we have only shown the curves of the electric field, as those of the magnetic field were always on top of the reference curves (dashed) computed from the exact solutions. 

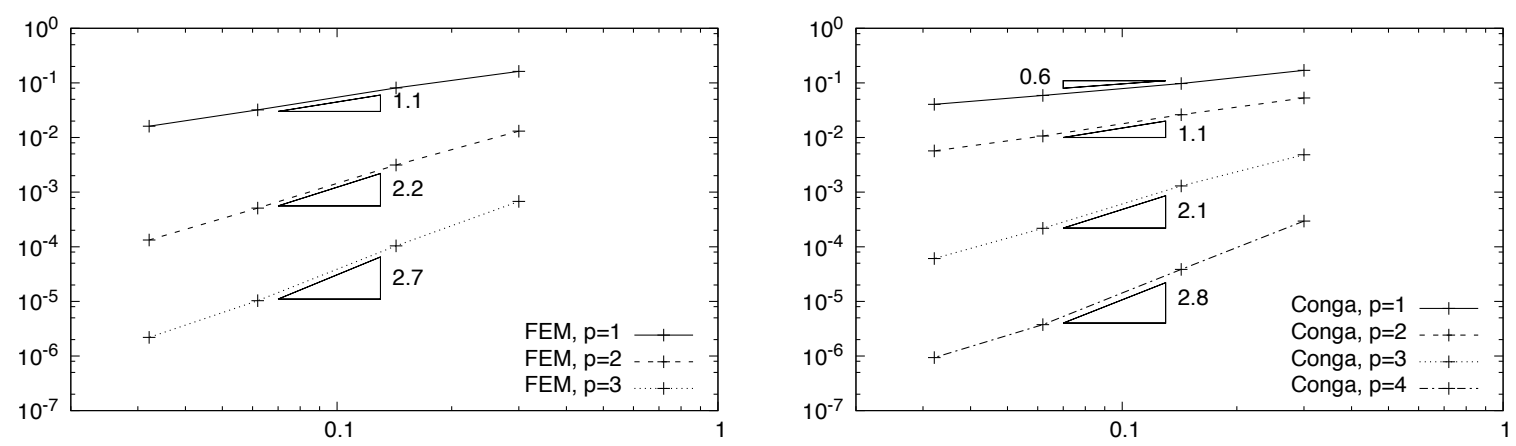

FiguRE 6.1. Convergence curves (relative errors vs. maximal triangle diameter $h$ ) for the Issautier problem with analytical source (6.1). Results obtained with the conforming FEM discretization are shown left, and those obtained with the non-conforming Conga discretization are shown right.
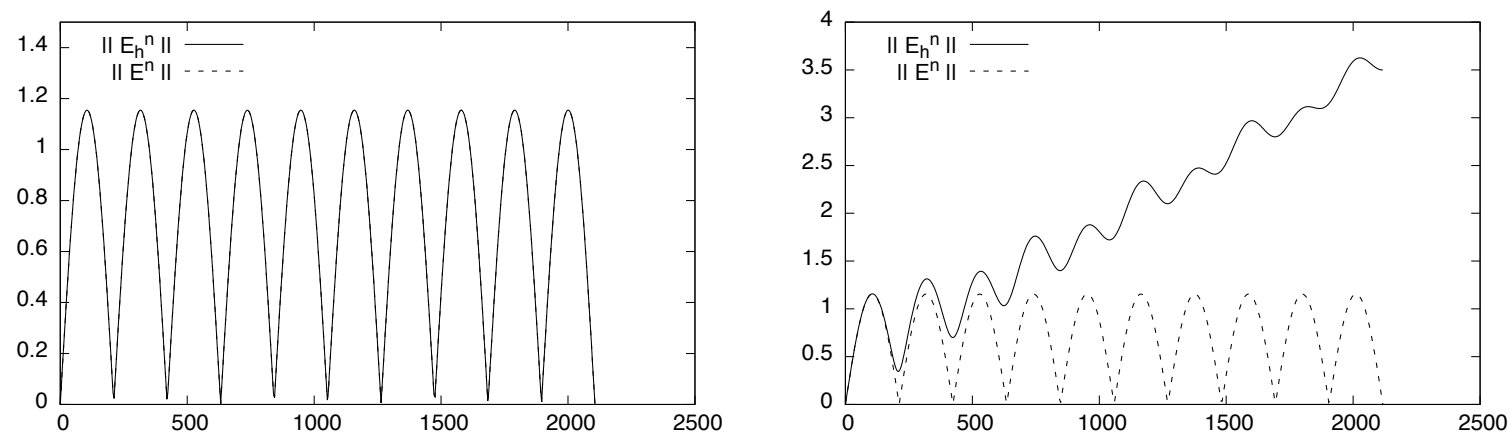

FiguRE 6.2. Evolution of the $L^{2}$ norm of the electric field for the Issautier problem. On the left plot the numerical solution is obtained by approximating the current density with a corrected projection on the fully discontinuous space $\tilde{V}_{h}^{2}$, see $(6.3)$, whereas on the right plot the current density is approximated using a standard $L^{2}$ projection (6.4). For comparison, the norm of the exact solution is shown in dashed lines (on the left plot it is on top of the solid line).

\subsection{A Vlasov-Maxwell problem: an academic diode test case}

Turning to the coupled FEM-PIC and Conga-PIC schemes, we use again the academic diode test case employed in [10] to test particle schemes coupled with Maxwell solvers with a strong Ampère law. Here the domain is a square $\Omega=[0,0.1 \mathrm{~m}]^{2}$ with metallic boundary $\Gamma_{M}=\{0,0.1 \mathrm{~m}\} \times[0,0.1 \mathrm{~m}]$ and absorbing boundary $\left.\Gamma_{A}=\right] 0,0.1 \mathrm{~m}[\times\{0,0.1 \mathrm{~m}\}$. On the left boundary a beam of electrons is steadily injected and accelerated by a constant external field which derives from the electric potential imposed on both the cathode ( $\phi_{\text {ext }}=0$ on the left boundary) and the anode $\left(\phi_{\text {ext }}=10^{5} \mathrm{~V}\right.$ on the right boundary). Due to the propagation of the beam into the domain (initially empty of charges) a self-consistent electromagnetic field develops and is added to this constant external field, and in turn the trajectories of the electrons are no longer straight lines. However this modification is of small relative amplitude and the resulting solution tends towards a smooth steady state, so that the convergence of the numerical approximations can be easily assessed. In Figure 6.3 we show the typical profile of the solution in the steady state regime (self-consistent electric field on the left and particles on the right), together with the mesh used in the simulations. 


\section{Compatible Maxwell solvers With Particles, II}

To avoid using expensive numerical quadratures in space we consider a coupling with point particles, as described in Section 5.4. The numerical algorithms tested here may then be seen as an extension of those proposed in [9] to the case of fully discontinuous elements.
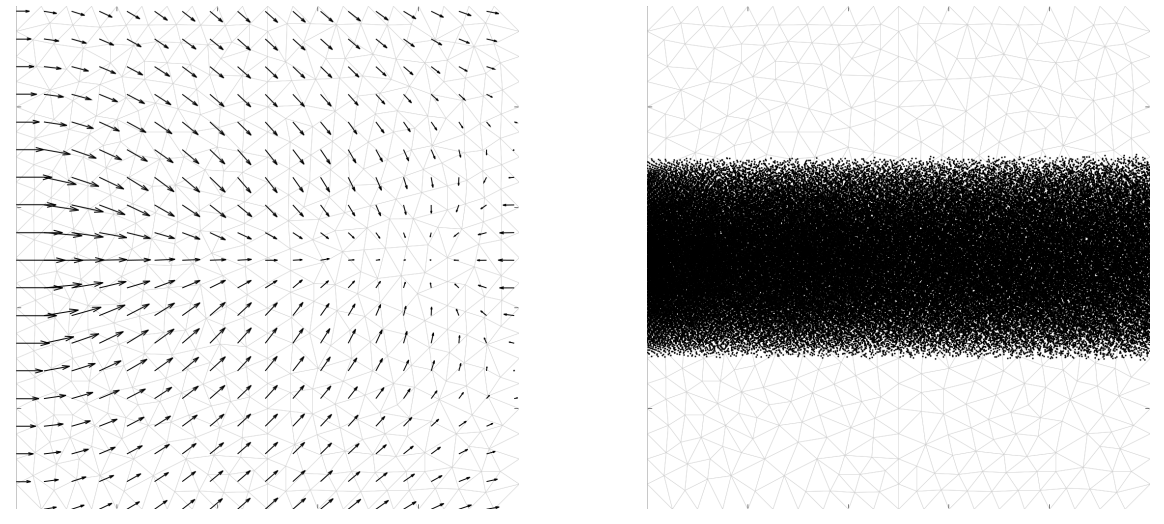

Figure 6.3. Academic beam test case. The self-consistent $\boldsymbol{E}$ field (left plot) and the numerical particles accelerated towards the right boundary (right plot) show the typical profile of the solution in the steady state regime. For the considered geometry the external field is constant $\boldsymbol{E}_{\text {ext }}=\left(-10^{6}, 0\right) \mathrm{Vm}^{-1}$.

To finally assess the numerical stability properties of the proposed FEM and Conga methods over long time ranges we plot in Figure 6.4 the profiles of several fields using a final time chosen so that the particles have travelled approximatively five diode lengths.

On the left column of Figure 6.4 we plot the profiles of the electro-magnetic field computed with the conforming FEM-PIC scheme (5.17) using a standard $L^{2}$ projection for the particle current, which consists in defining $\boldsymbol{J}_{h}^{n+1 / 2}:=P_{V_{h}^{2}} \boldsymbol{J}_{N}^{n+1 / 2}$ through products of the form (5.19). The stability of such a coupling is supported by Theorem 5.6, and indeed the numerical results show a very good preservation of the smooth steady state, both for the electric field (top and center row) and the magnetic field (bottom row). On the center and right columns of Figure 6.4 we then plot the fields computed with the non-conforming Conga-PIC scheme (5.18) using two different deposition methods for the current, similarly as what was done (starting from an analytical expression for $\boldsymbol{J}$ ) in Figure 6.2.

On the center column the DG current is obtained with a standard $L^{2}$ projection of the particle current, i.e., $\boldsymbol{J}_{h}^{n+1 / 2}:=P_{\tilde{V}_{h}^{2}} \boldsymbol{J}_{N}^{n+1 / 2}$, and on the right column it is defined as $\boldsymbol{J}_{h}^{n+1 / 2}:=\left(\mathcal{P}_{h}^{2}\right)^{*} \boldsymbol{J}_{N}^{n+1 / 2}$. Note that in practice this may be done either by computing terms of the form (5.19) and inverting the block-diagonal DG mass matrix of $\tilde{V}_{h}^{2}$, or by correcting locally the array of coefficients computed with the standard method, as described in Section 5.5. Again, the enhanced stability of the former coupling is supported by Theorem 5.6. This is clearly confirmed by our numerical simulation. Whereas the charge-conserving Conga scheme yields results comparable to the conforming method, the electric field resulting from the standard DG deposition scheme has erratic oscillations that grow linearly in time and reach, in the test done here, values about four times greater than the maximum amplitude of the correct solution.

\section{Conclusion}

In this series of papers we have provided a rigorous solution to the longstanding problem of chargeconserving coupling between general Maxwell solvers and particle methods, following the classical approach developped by plasma physicists over the last decades. Our stability analysis extends a recent work on compatible source approximation operators for pure Maxwell solvers, and it is based on 

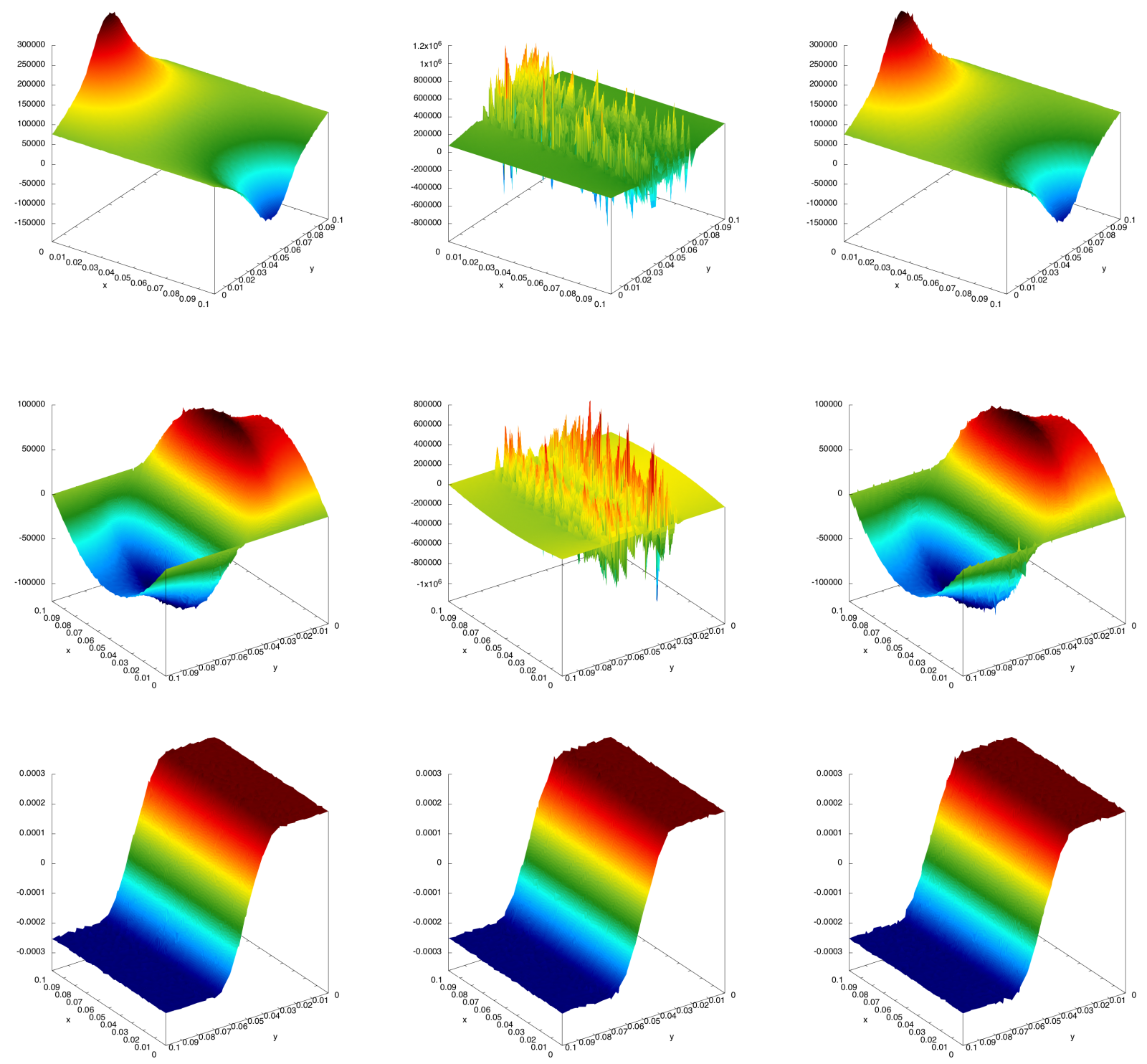

Figure 6.4. Academic beam test-case. Snapshots of the self-consistent fields ( $E_{x}$ on the top row, $E_{y}$ on the center row and $B$ on the bottom row) obtained by depositing the conservative current density carried by the particles with either the conforming FEMPIC scheme with standard $L^{2}$ projection for the particle current (left), the DG-PIC scheme with standard $L^{2}$ projection for the current (center) and the DG-PIC scheme with the corrected projection (6.3) for the particle current (right).

the notion of discrete de Rham structure. This abstract setting allows us to design charge-conserving deposition schemes for general conforming but also non-conforming Maxwell discretizations, thus offering an interesting alternative to divergence cleaning methods to stabilize Discontinuous Galerkin (DG) Particle-in-Cell solvers.

The framework of de Rham sequences also allows the choice of discretizing either the Ampère or the Faraday equation strongly, the other being handled by duality. In this paper we provided the 


\section{Compatible Maxwell solvers With Particles, II}

discrete framework for a strong Faraday equation and verified that it can be applied to a classical curl-conforming (Nédélec) Finite Element discretization, and also to a new hybrid non-conforming discretization based on broken Nédélec elements, having the advantage of avoiding global coupling. In $2 \mathrm{D}$ this discretization can be interpreted as a DG-PIC discretization with a corrected deposition scheme.

Numerical experiments using a pure Maxwell problem and a simple diode configuration allowed us to validate the theoretical stability of the proposed methods. Future studies should now address more elaborate test-cases to better understand the benefits of these structure-preserving and chargeconserving solvers with a strong Faraday law. Another open problem of interest is the study of discontinuous solvers based on different polynomial spaces, such as standard ones.

\section{Acknowledgements}

The authors would like to thank Stéphanie Salmon, Marie Mounier and Mathieu Lutz for stimulating discussions. They also acknowledge the anonymous referees for the questions and comments that helped clarify the presentation of this work.

\section{References}

[1] D.N. Arnold, R.S. Falk, and R. Winther. Finite element exterior calculus, homological techniques, and applications. Acta Numerica, 2006.

[2] D.N. Arnold, R.S. Falk, and R. Winther. Geometric decompositions and local bases for spaces of finite element differential forms. Computer Methods in Applied Mechanics and Engineering, 198(21):1660-1672, 2009.

[3] D.N. Arnold, R.S. Falk, and R. Winther. Finite element exterior calculus: from Hodge theory to numerical stability. Bull. Amer. Math. Soc.(NS), 47(2):281-354, 2010.

[4] D. Boffi, F. Brezzi, and M. Fortin. Mixed finite element methods and applications, volume 44 of Springer Series in Computational Mathematics. Springer, 2013.

[5] Daniele Boffi, Martin Costabel, Monique Dauge, and Leszek F Demkowicz. Discrete Compactness for the hp Version of Rectangular Edge Finite Elements. SIAM Journal on Numerical Analysis, 44(3):979-1004, 2006.

[6] A. Buffa and I. Perugia. Discontinuous Galerkin Approximation of the Maxwell Eigenproblem. SIAM Journal on Numerical Analysis, 44(5):2198-2226, January 2006.

[7] M. Campos Pinto. Constructing exact sequences on non-conforming discrete spaces. Comptes Rendus Mathematique, 354(7):691-696, 2016.

[8] M. Campos Pinto. Structure-preserving conforming and nonconforming discretizations of mixed problems. hal.archives-ouvertes.fr, February 2017.

[9] M. Campos Pinto, S. Jund, S. Salmon, and E. Sonnendrücker. Charge conserving FEM-PIC schemes on general grids. C.R. Mecanique, 342(10-11):570-582, 2014.

[10] M. Campos Pinto and E. Sonnendrücker. Compatible Maxwell solvers with particles I: conforming and non-conforming 2D schemes with a strong Ampère law. HAL preprint, 〈hal-01303852〉, 2016.

[11] M. Campos Pinto and E. Sonnendrücker. Gauss-compatible Galerkin schemes for time-dependent Maxwell equations. Mathematics of Computation, 2016.

[12] S. Depeyre and D. Issautier. A new constrained formulation of the Maxwell system. Rairo-Mathematical Modelling and Numerical Analysis-Modelisation Mathematique Et Analyse Numerique, 31(3):327-357, 1997. 


\section{Campos Pinto \& E. Sonnendrǘker}

[13] A. Ern and J.-L. Guermond. Finite Element Quasi-Interpolation and Best Approximation . 〈hal$01155412 \mathrm{v} 2\rangle, 2015$.

[14] L. Fezoui, S. Lanteri, S. Lohrengel, and S. Piperno. Convergence and stability of a discontinuous Galerkin time-domain method for the 3D heterogeneous Maxwell equations on unstructured meshes. ESAIM: Mathematical Modelling and Numerical Analysis, 39(6):1149-1176, November 2005.

[15] V. Girault and P.-A. Raviart. Finite Element Methods for Navier-Stokes Equations - Theory and Algorithms. Springer Series in Computational Mathematics. Springer-Verlag, Berlin, 1986.

[16] J.S. Hesthaven and T. Warburton. High-order nodal discontinuous Galerkin methods for the Maxwell eigenvalue problem. Philosophical Transactions of the Royal Society A: Mathematical, Physical and Engineering Sciences, 362(1816):493-524, March 2004.

[17] D. Issautier, F. Poupaud, J.-P. Cioni, and L. Fezoui. A 2-D Vlasov-Maxwell solver on unstructured meshes. In Third international conference on mathematical and numerical aspects of wave propagation, pages 355$371,1995$.

[18] G.B. Jacobs and J.S. Hesthaven. High-order nodal discontinuous Galerkin particle-in-cell method on unstructured grids. Journal of Computational Physics, 214(1):96-121, May 2006.

[19] C.G. Makridakis and P. Monk. Time-discrete finite element schemes for Maxwell's equations. RAIRO Modél Math Anal Numér, 29(2):171-197, 1995.

[20] P. Monk. An analysis of Nédélec's method for the spatial discretization of Maxwell's equations. Journal of Computational and Applied Mathematics, 47(1):101-121, 1993.

[21] J.-C. Nédélec. Mixed finite elements in $\mathbf{R}^{\mathbf{3}}$. Numerische Mathematik, 35(3):315-341, 1980.

[22] J.-C. Nédélec. A new family of mixed finite elements in $\mathbf{R}^{\mathbf{3}}$. Numerische Mathematik, 50(1):57-81, 1986.

[23] P.-A. Raviart and J.-M. Thomas. A mixed finite element method for 2nd order elliptic problems. In Mathematical aspects of finite element methods, Lecture Notes in Math., Vol. 606, pages 292-315. Springer, Berlin, 1977.

[24] A. Stock, J. Neudorfer, R. Schneider, C. Altmann, and C.-D. Munz. Investigation of the Purely Hyperbolic Maxwell System for Divergence Cleaning in Discontinuous Galerkin based Particle-In-Cell Methods. In COUPLED PROBLEMS 2011 IV International Conference on Computational Methods for Coupled Problems in Science and Engineering, 2011.

[25] J. Zhao. Analysis of finite element approximation for time-dependent Maxwell problems. Mathematics of Computation, 73(247):1089-1106, 2004. 